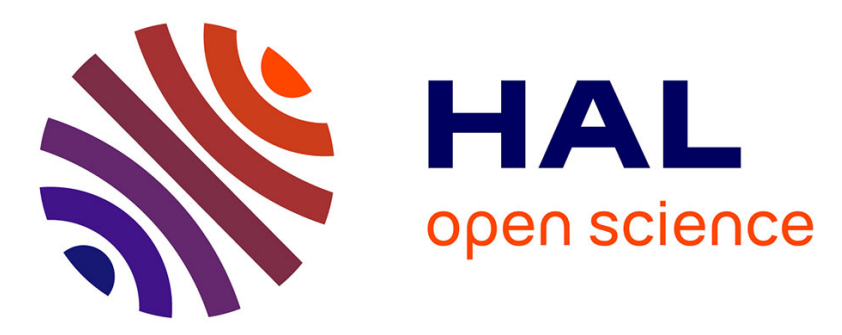

\title{
The Hodge-de Rham Laplacian and Lp-boundedness of Riesz transforms on non-compact manifolds
}

\author{
Peng Chen, Jocelyn Magniez, El Maati Ouhabaz
}

\section{To cite this version:}

Peng Chen, Jocelyn Magniez, El Maati Ouhabaz. The Hodge-de Rham Laplacian and Lp-boundedness of Riesz transforms on non-compact manifolds. Nonlinear Analysis: Theory, Methods and Applications, 2015, 125, pp.78-98. hal-01079425

\section{HAL Id: hal-01079425 \\ https://hal.science/hal-01079425}

Submitted on 1 Nov 2014

HAL is a multi-disciplinary open access archive for the deposit and dissemination of scientific research documents, whether they are published or not. The documents may come from teaching and research institutions in France or abroad, or from public or private research centers.
L'archive ouverte pluridisciplinaire HAL, est destinée au dépôt et à la diffusion de documents scientifiques de niveau recherche, publiés ou non, émanant des établissements d'enseignement et de recherche français ou étrangers, des laboratoires publics ou privés. 


\title{
RIESZ TRANSFORMS ON NON-COMPACT MANIFOLDS
}

\author{
PENG CHEN, JOCELYN MAGNIEZ AND EL MAATI OUHABAZ
}

ABSTRACT. Let $M$ be a complete non-compact Riemannian manifold satisfying the doubling volume property as well as a Gaussian upper bound for the corresponding heat kernel. We study the boundedness of the Riesz transform $d \Delta^{-\frac{1}{2}}$ on both Hardy spaces $H^{p}$ and Lebesgue spaces $L^{p}$ under two different conditions on the negative part of the Ricci curvature $R^{-}$. First we prove that if $R^{-}$is $\alpha$-subcritical for some $\alpha \in[0,1)$, then the Riesz transform $d^{*} \vec{\Delta}^{-\frac{1}{2}}$ on differential 1-forms is bounded from the associated Hardy space $H_{\vec{\Delta}}^{p}\left(\Lambda^{1} T^{*} M\right)$ to $L^{p}(M)$ for all

$p \in[1,2]$. As a consequence, $d \Delta^{-\frac{1}{2}}$ is bounded on $L^{p}$ for all $p \in\left(1, p_{0}\right)$ where $p_{0}>2$ depends on $\alpha$ and the constant appearing in the doubling property. Second, we prove that if

$$
\int_{0}^{1}\left\|\frac{\left|R^{-}\right|^{\frac{1}{2}}}{v(\cdot, \sqrt{t})^{\frac{1}{p_{1}}}}\right\|_{p_{1}} \frac{d t}{\sqrt{t}}+\int_{1}^{\infty}\left\|\frac{\left|R^{-}\right|^{\frac{1}{2}}}{v(\cdot, \sqrt{t})^{\frac{1}{p_{2}}}}\right\|_{p_{2}} \frac{d t}{\sqrt{t}}<\infty,
$$

for some $p_{1}>2$ and $p_{2}>3$, then the Riesz transform $d \Delta^{-\frac{1}{2}}$ is bounded on $L^{p}$ for all $1<p<p_{2}$. In the particular case where $v(x, r) \geq C r^{D}$ for all $r \geq 1$ and $\left|R^{-}\right| \in L^{D / 2-\eta} \cap L^{D / 2+\eta}$ for some $\eta>0$, then $d \Delta^{-\frac{1}{2}}$ is bounded on $L^{p}$ for all $1<p<D$.

Furthermore, we study the boundedness of the Riesz transform of Schrödinger operators $A=\Delta+V$ on $L^{p}$ for $p>2$ under conditions on $R^{-}$and the potential $V$. We prove both positive and negative results on the boundedness of $d A^{-\frac{1}{2}}$ on $L^{p}$.

\section{INTRODUCTION}

Let $(M, g)$ be a complete non-compact Riemannian manifold and let $\rho$ be the geodesic distance and $\mu$ be the Riemannian measure associated with the metric $g$. Assume that $M$ satisfies the doubling volume property, that is, there exists a constant $C>0$ such that

$$
v(x, 2 r) \leq C v(x, r) \text { for all } x \in M, r \geq 0,
$$

where $v(x, r)$ denotes the volume of the ball $B(x, r)$ of center $x$ and radius $r$. This property is equivalent to the following one. There exist constants $C>0$ and $D>0$ such that

$$
v(x, \lambda r) \leq C \lambda^{D} v(x, r) \text { for all } x \in M, r \geq 0, \lambda \geq 1 .
$$

In the sequel we will be interested in the smallest possible $D$ for which (1.1) holds. For convenience we call $D$ "the" doubling dimension or the homogeneous dimension.

Date: October 23, 2014.

2000 Mathematics Subject Classification. 42B20 42B30 58J35 47F05.

Key words and phrases. The Riesz transform, Riemannian manifold, the Hodge-de Rham Laplacian, Hardy spaces, Schrödinger operators, the heat kernel.

The research of the authors was partially supported by the ANR project HAB, ANR-12-BS01-0013-02. 
Let $\Delta$ be the non-negative Laplace-Beltrami operator on $M$ and $p_{t}(x, y)$ the corresponding heat kernel, i.e., the integral kernel of the semigroup $e^{-t \Delta}$. We assume that $p_{t}(x, y)$ satisfies a Gaussian upper bound

$$
p_{t}(x, y) \leq C v(x, \sqrt{t})^{-1} e^{-c \frac{\rho^{2}(x, y)}{t}} \text { for all } t>0, x, y \in M,
$$

where $c, C>0$ are constants. The validity of (1.2) has been intensively studied in the literature.

We consider the Riesz transform $d \Delta^{-\frac{1}{2}}$. Integration by parts shows that $d \Delta^{-\frac{1}{2}}$ is bounded from $L^{2}(M)$ to $L^{2}\left(\Lambda^{1} T^{*} M\right)$, where $\Lambda^{1} T^{*} M$ denotes the space of differential 1-forms. We address the problem whether the Riesz transform $d \Delta^{-\frac{1}{2}}$ could be extended to a bounded operator from $L^{p}(M)$ to $L^{p}\left(\Lambda^{1} T^{*} M\right)$ for $p \neq 2$.

Under the assumptions (1.1) and (1.2), it was proved by Coulhon and Duong [12] that $d \Delta^{-\frac{1}{2}}$ is bounded from $L^{p}(M)$ to $L^{p}\left(\Lambda^{1} T^{*} M\right)$ for all $p \in(1,2]$. They also gave a counter-example which shows that (1.1) and (1.2) are not sufficient in the case $p>2$. So additional assumptions are needed. Many works have been devoted to this problem.

Under Li-Yau estimates, or equivalently under the doubling condition and a $L^{2}$ Poincaré inequality, Auscher and Coulhon [2] proved that there exists $\epsilon>0$ such that $d \Delta^{-\frac{1}{2}}$ is bounded on $L^{p}$ for all $2 \leq p<2+\epsilon$. In the same setting, Auscher, Coulhon, Duong and Hofmann [3] found an equivalence between the boundedness of the Riesz transform on $L^{p}$ for $p>2$ and the gradient estimate $\left\|d e^{-t \Delta}\right\|_{p-p} \leq C / \sqrt{t}$ for the corresponding semigroup on $L^{p}$.

Bakry [7] proved that if the manifold has a non-negative Ricci curvature, then $d \Delta^{-\frac{1}{2}}$ is bounded on $L^{p}$ for all $p \in(1, \infty)$.

Another idea to treat the case $p>2$ is by duality. By the commutation formula $\vec{\Delta} d=d \Delta$, we are then interested in the Riesz transform $d^{*} \vec{\Delta}-\frac{1}{2}$ where $\vec{\Delta}=d d^{*}+d^{*} d$ is the Hodge-de Rham Laplacian on differential 1-forms. The boundedness of $d^{*} \vec{\Delta}^{-\frac{1}{2}}$ on $L^{q}$ for some $q \in(1,2)$ implies the boundedness of $d \Delta^{-\frac{1}{2}}$ on $L^{p}$ where $\frac{1}{p}+\frac{1}{q}=1$. This strategy was used in Coulhon and Duong [13] by looking at the heat kernel on differential forms. They also made an interesting connection between boundedness of the Riesz transform and Littlewood-Paley-Stein inequalities.

Let us recall the Böchner formula

$$
\vec{\Delta}=\nabla^{*} \nabla+R^{+}-R^{-}
$$

where $R^{+}$and $R^{-}$are respectively the positive and negative part of the Ricci curvature and $\nabla$ denotes the Levi-Civita connection on $M$. We use this formula to look at $\vec{\Delta}$ as a Schrödinger operator on 1-forms and then to bring known techniques for the Riesz transforms of Schrödinger operators on functions and try to adapt them to this setting. We note however that the boundedness of the Riesz transform of a Schrödinger operator is a delicate task even in the Euclidean setting. See Assaad and Ouhabaz [6] and also the last sections of the present paper.

First, we assume that the negative part $R^{-}$is $\alpha$-subcritical for some $\alpha \in[0,1)$, that is

$$
0 \leq\left(R^{-} \omega, \omega\right) \leq \alpha(H \omega, \omega):=\alpha\left(\left(\nabla^{*} \nabla+R^{+}\right) \omega, \omega\right) \text { for all } \omega \in C_{c}^{\infty}\left(\Lambda^{1} T^{*} M\right),
$$

where $(\cdot, \cdot)$ is the inner product in $L^{2}\left(\Lambda^{1} T^{*} M\right)$. Then we prove that $d^{*} \vec{\Delta}^{-\frac{1}{2}}$ is bounded from $H_{\vec{\Delta}}^{1}\left(\Lambda^{1} T^{*} M\right)$ to $L^{1}(M)$ where $H_{\vec{\Delta}}^{1}\left(\Lambda^{1} T^{*} M\right)$ is the Hardy spaces associated with the operator $\vec{\Delta}$, see Section 2 for details and definitions. By interpolation, we obtain that $d^{*} \vec{\Delta}-\frac{1}{2}$ is bounded from $L^{p}\left(\Lambda^{1} T^{*} M\right)$ to $L^{p}(M)$ for all $p \in(1,2]$ if $D \leq 2$ and all $p \in\left(p_{0}^{\prime}, 2\right]$ if $D>2$ where 
$p_{0}:=\frac{2 D}{(D-2)(1-\sqrt{1-\alpha})}$. The latter result was proved recently by Magniez [24]. As a corollary, the Riesz transform $d \Delta^{-\frac{1}{2}}$ is bounded from $L^{p}(M)$ to $L^{p}\left(\Lambda^{1} T^{*} M\right)$ for all $p \in(1, \infty)$ if $D \leq 2$ and all $p \in\left(1, p_{0}\right)$ if $D>2$.

The above $L^{p}$-boundedness result is not sharp in general. Note that if $\alpha$ is close to 1 and $D$ is large, then $p_{0}$ is close to 2. In [14], Devyver proved that if $M$ satisfies a Sobolev inequality together with the additional assumption that balls of large radius have a polynomial volume growth, that is $c r^{D} \leq v(x, r) \leq C r^{D}$ for $r \geq 1$, and $R^{-} \in L^{\frac{D}{2}-\varepsilon} \cap L^{\infty}$ for some $\varepsilon>0$, then the Riesz transform $d \Delta^{-\frac{1}{2}}$ is bounded from $L^{p}(M)$ to $L^{p}\left(\Lambda^{1} T^{*} M\right)$ for all $p \in(1, D)$.

Our aim is to get a similar result under the doubling condition for the volume without assuming the Sobolev inequality. We suppose that the negative part of the Ricci curvature $R^{-}$ satisfies

$$
\left\|R^{-}\right\|_{v o l}:=\int_{0}^{1}\left\|\frac{\left|R^{-}\right|^{\frac{1}{2}}}{v(\cdot, \sqrt{t})^{\frac{1}{p_{1}}}}\right\|_{p_{1}} \frac{d t}{\sqrt{t}}+\int_{1}^{\infty}\left\|\frac{\left|R^{-}\right|^{\frac{1}{2}}}{v(\cdot, \sqrt{t})^{\frac{1}{p_{2}}}}\right\|_{p_{2}} \frac{d t}{\sqrt{t}}<\infty,
$$

for some $p_{1}, p_{2}>2$. We prove that if $3<p_{2} \leq D$ then the Riesz transform $d \Delta^{-\frac{1}{2}}$ is bounded on $L^{p}$ for all $1<p<p_{2}$. In the particular case where the volume of balls has polynomial lower bound, $v(x, r) \geq C r^{D}$, then our condition $\left\|R^{-}\right\|_{v o l}<\infty$ is satisfied if $R^{-} \in L^{\frac{D}{2}-\eta} \cap L^{\frac{D}{2}+\eta}$ for some $\eta>0$. In this situation, $p_{2}$ can be chosen to be any number smaller than $D$ and our result shows that $d \Delta^{-\frac{1}{2}}$ is bounded on $L^{p}$ for all $1<p<D$. This latter result recovers and extends a result due to Devyver [14] who assumes $R^{-} \in L^{\frac{D}{2}-\eta} \cap L^{\infty}$. Let us also mention recent results by Carron [10] who proved in particular that if the negative part of the Ricci curvature has at most a quadratic growth and the volume satisfies a reverse doubling condition with a "dimension" $\nu$, then $d \Delta^{-\frac{1}{2}}$ is bounded on $L^{p}$ for $p \in(1, \nu)$.

In the last two sections of the paper we consider the Riesz transform of Schrödinger operators. Let $A=\Delta+V$ with signed potential $V=V^{+}-V^{-}$. Similarly to our first result, we assume that $V^{+} \in L_{l o c}^{1}$ and $V^{-}$satisfies $\alpha$-subcritical condition for some $\alpha \in[0,1)$ :

$$
\int_{M} V^{-} u^{2} d \mu \leq \alpha\left[\int_{M}|\nabla u|^{2} d \mu+\int_{M} V^{+} u^{2} d \mu\right] \text { for all } u \in W^{1,2}(M) .
$$

Under this assumption on the potential $V$, we prove that the associated Riesz transform $d A^{-\frac{1}{2}}$ is bounded from $H_{A}^{1}(M)$ to $L^{1}\left(\Lambda^{1} T^{*} M\right)$. By interpolation we obtain that $d A^{-\frac{1}{2}}$ is bounded on $L^{p}(M)$ for all $p \in(1,2]$ if $D \leq 2$ and all $p \in\left(p_{0}^{\prime}, 2\right]$ if $D>2$, where again $p_{0}:=\frac{2 D}{(D-2)(1-\sqrt{1-\alpha})}$. The latter result is proved by Assaad and Ouhabaz [6] by a different approach. For $p>2$, we assume in addition that the negative part of the Ricci curvature $R^{-}$satisfies (1.4) and also $V$ satisfies (1.4) that is

$$
\int_{0}^{1}\left\|\frac{|V|^{\frac{1}{2}}}{v(\cdot, \sqrt{t})^{\frac{1}{p_{1}}}}\right\|_{p_{1}} \frac{d t}{\sqrt{t}}+\int_{1}^{\infty}\left\|\frac{|V|^{\frac{1}{2}}}{v(\cdot, \sqrt{t})^{\frac{1}{p_{2}}}}\right\|_{p_{2}} \frac{d t}{\sqrt{t}}<\infty .
$$

Then we prove that $d A^{-\frac{1}{2}}$ is bounded on $L^{p}$ for all $p_{0}^{\prime}<p<\frac{p_{0} r}{p_{0}+r}$ where $r=\inf \left(p_{1}, p_{2}\right)$. In the particular case where the volume $v(x, r)$ has polynomial growth and $V^{-}=0$, our result implies that $d A^{-\frac{1}{2}}$ is bounded on $L^{p}$ for all $1<p<D$ provided $V \in L^{\frac{D}{2}-\eta} \cap L^{\frac{D}{2}+\eta}$ for some $\eta>0$. In the last section we prove that the interval $(1, D)$ cannot be improved in general. 
More precisely, we assume that the manifold $M$ satisfies the Poincaré inequality, the doubling condition (1.1) with the doubling constant $D$ and that there exists a positive bounded function $\phi$ such that $A \phi=0$. We then prove that if the Riesz transform $d A^{-\frac{1}{2}}$ is bounded on $L^{p}$ for some $p>D$, then $V=0$. A similar result was proved by Guillarmou and Hassell [19] on complete noncompact and asymptotically conic Riemannian manifold assuming $V$ is smooth and sufficiently vanishing at infinity. In particular this is satisfied on the Euclidean space $\mathbb{R}^{n}$ with a smooth and compactly supported potential $V$.

Throughout, the symbols " $c$ " and " $C$ " will denote (possibly different) constants that are independent of the essential variables.

\section{HARDY SPACES ASSOCIATED With SELF-ADJOINT OPERATORS}

In this section, we recall Hardy spaces $H_{L}^{p}$ associated with a given operator $L$ on manifolds. The operator $L$ is either acting on functions or on differential 1-forms. These Hardy spaces have been studied by several authors, see for example [1, 4, 5, 15, 21, 22, 23].

Let $(X, \rho, \mu)$ be a metric measured space satisfying the doubling condition (1.1). We denote by $T X$ a smooth vector bundle over $X$ with scalar product $(\cdot, \cdot)_{x}$. For $f(x) \in T_{x} X$ we put $|f(x)|_{x}^{2}=(f(x), f(x))_{x}$. To simplify the notation we will write $|\cdot|$ instead of $|\cdot|_{x}$. In the next sections of this paper, $X$ will be a complete non-compact Riemannian manifold.

First, we recall the definitions of the finite speed propagation property and the DaviesGaffney estimates for semigroup. For $r>0$, we set

$$
\mathcal{D}_{r}:=\{(x, y) \in X \times X: \rho(x, y) \leq r\} .
$$

Given an operator $T$ on $L^{2}(T X)$, we write

$$
\operatorname{supp} K_{T} \subseteq \mathcal{D}_{r}
$$

if $\left\langle T f_{1}, f_{2}\right\rangle=0$ for all $f_{k} \in C(T X)$ with $\operatorname{supp} f_{k} \subseteq B\left(x_{k}, r_{k}\right)$ for $k=1,2$ and $r_{1}+r_{2}+r<$ $\rho\left(x_{1}, x_{2}\right)$. If $T$ is an integral operator with kernel $K_{T}$, then (2.1) has the usual meaning $K_{T}(x, y)=0$ for a.e. $(x, y) \in X \times X$ with $\rho(x, y)>r$.

Definition 2.1. Given a non-negative self-adjoint operator $L$ on $L^{2}(T X)$. One says that the operator $L$ satisfies the finite speed propagation property if

$$
\operatorname{supp} K_{\cos (t \sqrt{L})} \subseteq \mathcal{D}_{t} \text { for all } t \geq 0 .
$$

Definition 2.2. One says that the semigroup $\left\{e^{-t L}\right\}_{t>0}$ generated by (minus) $L$ satisfies the Davies-Gaffney estimates if there exist constants $C, c>0$ such that for all open subsets $U_{1}, U_{2} \subset X$ and all $t>0$,

$$
\left|\left\langle e^{-t L} f_{1}, f_{2}\right\rangle\right| \leq C \exp \left(-\frac{\operatorname{dist}\left(U_{1}, U_{2}\right)^{2}}{c t}\right)\left\|f_{1}\right\|_{L^{2}(T X)}\left\|f_{2}\right\|_{L^{2}(T X)},
$$

for every $f_{i} \in L^{2}(T X)$ with supp $f_{i} \subset U_{i}, i=1,2$, where $\operatorname{dist}\left(U_{1}, U_{2}\right):=\inf _{x \in U_{1}, y \in U_{2}} \rho(x, y)$.

The following result is taken from [25, Theorem 2].

Proposition 2.3. Let $L$ be a non-negative self-adjoint operator acting on $L^{2}(T X)$. Then the finite speed propagation property (FS) and Davies-Gaffney estimates (DG) are equivalent. 
Next we recall the definition of Hardy spaces associated with self-adjoint operators. Assume that the operator $L$ satisfies the Davies-Gaffney estimates (DG). Following [4, 5, 15, 21] one can define the $L^{2}$ adapted Hardy space by

$$
H^{2}(T X):=\overline{R(L)}
$$

that is, the closure of the range of $L$ in $L^{2}(T X)$. Then $L^{2}(T X)$ is the orthogonal sum of $H^{2}(T X)$ and the null space $N(L)$. Consider the following quadratic functional associated to $L:$

$$
S_{K} f(x):=\left(\int_{0}^{\infty} \int_{\rho(x, y)<t}\left|\left(t^{2} L\right)^{K} e^{-t^{2} L} f(y)\right|^{2} \frac{d \mu(y)}{v(x, t)} \frac{d t}{t}\right)^{\frac{1}{2}},
$$

where $x \in X, f \in L^{2}(T X)$ and $K$ is a natural number. For each $K \geq 1$ and $0<p<\infty$, we now define

$$
D_{K, p}:=\left\{f \in H^{2}(T X): S_{K} f \in L^{p}(X)\right\} .
$$

Definition 2.4. Let $L$ be a non-negative self-adjoint operator on $L^{2}(T X)$ satisfying the DaviesGaffney estimates (DG).

(i) For each $p \in(0,2]$, the Hardy space $H_{L}^{p}(T X)$ associated with $L$ is the completion of the space $D_{1, p}$ with respect to the norm

$$
\|f\|_{H_{L}^{p}(T X)}:=\left\|S_{1} f\right\|_{L^{p}(X)}
$$

(ii) For each $p \in(2, \infty)$, the Hardy space $H_{L}^{p}(T X)$ associated with $L$ is the completion of the space $D_{K_{0}, p}$ in the norm

$$
\|f\|_{H_{L}^{p}(T X)}:=\left\|S_{K_{0}} f\right\|_{L^{p}(X)}, \quad \text { where } K_{0}=\left[\frac{D}{4}\right]+1
$$

It can be verified that the dual of $H_{L}^{p}(T X)$ is $H_{L}^{p^{\prime}}(T X)$, with $\frac{1}{p}+\frac{1}{p}^{\prime}=1$ (see Proposition 9.4 of [21]). We also have complex interpolation and Marcinkiewicz-type interpolation results between $H_{L}^{p}(T X)$ for $1 \leq p \leq 2$ (see Proposition 9.5 and Theorem 9.7 [21]). Although the above results in [21] are stated and proved on $X$ and not on $T X$, the whole machinery developed there works in the context of Hardy spaces over $T X$.

Note that if we only assume Davies-Gaffney estimates on the heat kernel of $L$, for $1<p<\infty$, $p \neq 2, H_{L}^{p}(T X)$ may or may not coincide with the space $L^{p}(T X)$. For the relation of $H_{L}^{p}(T X)$ and $L^{p}(T X)$ for $1<p \leq 2$, we have the following proposition.

Proposition 2.5. Let $L$ be an injective, nonnegative self-adjoint operator on $L^{2}(T X)$ satisfying the finite propagation speed property (FS) and general $\left(p_{0}, 2\right)$-Davies-Gaffney estimates

$\left(\mathrm{DG}_{\mathrm{p}_{0}}\right) \quad\left\|\chi_{B(x, t)} e^{-t^{2} L} \chi_{B(y, t)}\right\|_{p_{0} \rightarrow 2} \leq C v(x, t)^{\frac{1}{2}-\frac{1}{p}} \exp \left(-c \rho(x, y)^{2} / t^{2}\right)$

for some $p_{0}$ with $1 \leq p_{0} \leq 2$. Then for each $p$ with $p_{0}<p \leq 2$, the Hardy space $H_{L}^{p}(T X)$ and the Lebesgue space $L^{p}(T X)$ coincide and their norms are equivalent.

Proof. This proof is the same as for Hardy space $H_{L}^{p}(X)$ (see [23, Proposition 9.1(v)] and [1]). For more details, see also [29, Theorem 4.19].

We denote by $\mathcal{D}(L)$ the domain of the operator $L$. The following definition of atoms of Hardy spaces associated with operators was introduced in [21]. 
Definition 2.6. Let $M$ be a positive integer. A function $a \in L^{2}(T X)$ is called a $(1,2, M)$-atom associated with $L$ if there exist a function $b \in \mathcal{D}\left(L^{M}\right)$ and a ball $B$ such that

(i) $a=L^{M} b$,

(ii) $\operatorname{supp} L^{k} b \subset B, k=0,1, \ldots, M$,

(iii) $\left\|\left(r_{B}^{2} L\right)^{k} b\right\|_{L^{2}} \leq r_{B}^{2 M} v(B)^{-\frac{1}{2}}, k=0,1, \ldots, M$, where $v(B)$ is the volume of the ball $B$.

We can define the atomic Hardy space $H_{L, a t, M}^{1}(T X)$ as follows. First, we say that $f=\sum \lambda_{j} a_{j}$ is an atomic $(1,2, M)$-representation if $\left\{\lambda_{j}\right\}_{j=0}^{\infty} \in \ell^{1}$, each $a_{j}$ is a $(1,2, M)$-atom and the sum converges in $L^{2}(T X)$. Then set

$$
\mathbb{H}_{L, a t, M}^{1}(T X):=\{f: f \text { has an atomic }(1,2, M) \text {-representation }\},
$$

with the norm given by

$$
\|f\|_{\mathbb{H}_{L, a t, M}^{1}(T X)}:=\inf \left\{\sum_{j=0}^{\infty}\left|\lambda_{j}\right|: f=\sum_{j=0}^{\infty} \lambda_{j} a_{j} \text { is an atomic }(1,2, M) \text {-representation }\right\} .
$$

The space $H_{L, a t, M}^{1}(T X)$ is then defined as the completion of $\mathbb{H}_{L, a t, M}^{1}(T X)$ with respect to this norm. According to [21, Theorem 2.5], if $M>D / 4$ and $L$ satisfies Davies-Gaffney estimate (DG), then Hardy space $H_{L}^{1}(T X)$ coincides with the atomic Hardy space $H_{L, a t, M}^{1}(T X)$ and their norms are equivalent.

\section{Boundedness of Riesz TRANSFORMS ON HARDY SPACES OF FORMS}

To prove the boundedness of the Riesz transform on Hardy spaces associated with self-adjoint operators on forms, we need the following lemma.

Lemma 3.1. Assume that $T$ is a non-negative sublinear operator and bounded from $L^{2}(T X)$ to $L^{2}(X)$. Also assume that for every $(1,2, M)$-atom a, we have

$$
\|T a\|_{L^{1}(X)} \leq C
$$

with constant $C$ independent of $a$. Then $T$ is bounded from $H_{L}^{1}(T X)$ to $L^{1}(X)$.

Proof. For the proof, we refer the reader to [21, Lemma 4.3 and Proposition 4.13].

We now state a criterion that allows to derive estimates on Hardy spaces $H_{L}^{p}(T X)$. It is already stated in [16, Theorem 3.1] for spectral multipliers $T=m(L)$ on $H_{L}^{p}(X)$. We show that the arguments there are valid for a general linear operator $T$ which is bounded on $L^{2}$. We do not require the commutation of $T$ with the semigroup $e^{-t L}$.

Let $U_{j}(B)=2^{j+1} B \backslash 2^{j} B=U_{j}$ when $j \geq 2$ and $U_{1}(B)=4 B$.

Lemma 3.2. Let $L$ be a non-negative self-adjoint operator acting on $L^{2}(T X)$ and satisfying the Davies-Gaffney estimates (DG). Let $T$ be a linear operator which is bounded from $L^{2}(T X)$ to $L^{2}(X)$. Assume that there exist constants $M \geq 1, s>D / 2$ and $C>0$ such that for every $j=1,2, \ldots$,

$$
\left\|T\left(I-e^{-r^{2} L}\right)^{M} f\right\|_{L^{2}\left(U_{j}(B)\right)} \leq C 2^{-j s}\|f\|_{L^{2}(B)}
$$

for every ball $B$ with radius $r$ and for all $f \in L^{2}(T X)$ with supp $f \subset B$. Then the operator $T$ extends to a bounded operator from $H_{L}^{1}(T X)$ to $L^{1}(X)$. 
Proof. Let $a=L^{M} b$ be a $(1,2, M)$-atom. By Lemma 3.1, it is enough to prove that

$$
\|T a\|_{L^{1}(X)} \leq C
$$

with constant $C$ independent of the atom $a$.

Denote $B:=B(x, r)$ the ball containing the support of the atom $a$. We have

$$
\|T a\|_{L^{1}(X)} \leq \sum_{j=1}^{\infty}\|T a\|_{L^{1}\left(U_{j}(B)\right)}
$$

Note that by Hölder's inequality and $L^{2}$-boundedness of the operator $T$

$$
\|T a\|_{L^{1}(4 B)} \leq v(4 B)^{\frac{1}{2}}\|T a\|_{L^{2}(X)} \leq C v(B)^{\frac{1}{2}}\|a\|_{L^{2}(T X)} .
$$

By (iii) of Definition 2.6, $\|a\|_{L^{2}} \leq v(B)^{-\frac{1}{2}}$ and thus

$$
\|T a\|_{L^{1}(4 B)} \leq C v(B)^{\frac{1}{2}} v(B)^{-\frac{1}{2}} \leq C .
$$

Then we only need to prove that there exist some constants $\varepsilon>0$ and $C>0$ independent of the atom $a$ such that

$$
\|T a\|_{L^{1}\left(U_{j}(B)\right)} \leq C 2^{-j \varepsilon}
$$

for $j=2,3, \ldots$

Following (8.7) and (8.8) in [22] or (3.5) in [16], we write

$$
\begin{aligned}
I & =2\left(r^{-2} \int_{r}^{\sqrt{2} r} t d t\right) \cdot I \\
& =2 r^{-2} \int_{r}^{\sqrt{2} r} t\left(I-e^{-t^{2} L}\right)^{M} d t+\sum_{\alpha=1}^{M} C_{j, M} r^{-2} \int_{r}^{\sqrt{2} r} t e^{-j t^{2} L} d t
\end{aligned}
$$

where $C_{\alpha, M}$ are some constants depending only on $\alpha$ and $M$ only. Using the fact that $\partial_{t} e^{-\alpha t^{2} L}=$ $-2 \alpha t L e^{-\alpha t^{2} L}$ and applying the procedure $M$ times, we have for every function $f$ on $T X$,

$$
\begin{aligned}
f= & 2^{M}\left(r^{-2} \int_{r}^{\sqrt{2} r} t\left(I-e^{-t^{2} L}\right)^{M} d t\right)^{M} f \\
+ & \sum_{\beta=1}^{M} r^{-2 \beta}\left(I-e^{-r^{2} L}\right)^{\beta}\left(r^{-2} \int_{r}^{\sqrt{2} r} t\left(I-e^{-t^{2} L}\right)^{M} d t\right)^{M-\beta} \sum_{\alpha=1}^{(2 M-1) \beta} C_{\beta, \alpha, M} e^{-\alpha r^{2} L} L^{-\beta} f \\
:= & \sum_{\beta=0}^{M-1} r^{-2 \beta} r^{-2} \int_{r}^{\sqrt{2} r} t F_{\beta, M, r}(L)\left(I-e^{-t^{2} L}\right)^{M} L^{-\beta} f d t \\
& +r^{-2 M} F_{M, M, r}(L)\left(I-e^{-r^{2} L}\right)^{M} L^{-M} f
\end{aligned}
$$

where

$$
F_{\beta, M, r}(L)=\left(I-e^{-r^{2} L}\right)^{\beta}\left(r^{-2} \int_{r}^{\sqrt{2} r} t\left(I-e^{-t^{2} L}\right)^{M} d t\right)^{M-\beta-1} \sum_{\alpha=1}^{(2 M-1) \beta} C_{\beta, \alpha, M} e^{-\alpha r^{2} L}
$$


for $0 \leq \beta \leq M-1$ and

$$
F_{M, M, r}(L)=\sum_{\alpha=1}^{(2 M-1) M} C_{M, \alpha, M} e^{-\alpha r^{2} L} .
$$

It follows from the Davies-Gaffney estimates that the operator $F_{\beta, M, r}(L), \beta=0,1, \cdots, M$, satisfies $L^{2}$ off-diagonal estimates, which means that there exist some constants $c, C>0$ such that

$$
\left\|F_{\beta, M, r}(L) f\right\|_{L^{2}\left(U_{j}(B)\right)} \leq C e^{-c 4^{|j-i|}}\|f\|_{L^{2}\left(U_{i}(B)\right)} .
$$

For the details, see [16, pp. 307-309].

By (i) of Definition 2.6, $a=L^{M} b$. Then applying (3.5), we have

$$
\begin{aligned}
T a= & \sum_{\beta=0}^{M-1} r^{-2 \beta} r^{-2} \int_{r}^{\sqrt{2} r} t T\left(I-e^{-t^{2} L}\right)^{M} F_{\beta, M, r}(L) L^{M-\beta} b d t \\
& +r^{-2 M} T\left(I-e^{-r^{2} L}\right)^{M} F_{M, M, r}(L) b .
\end{aligned}
$$

Then by Hölder's inequality

$$
\begin{aligned}
& \|T a\|_{L^{1}\left(U_{j}(B)\right)} \\
& \leq C v\left(U_{j}(B)\right)^{\frac{1}{2}} \sum_{\beta=0}^{M} r^{-2 \beta} \sup _{t \in[r, \sqrt{2} r]}\left\|T\left(I-e^{-t^{2} L}\right)^{M} F_{\beta, M, r}(L) L^{M-\beta} b\right\|_{L^{2}\left(U_{j}(B)\right)} .
\end{aligned}
$$

For $t \in[r, \sqrt{2} r]$, let $B^{t}:=B(x, t)$. Then

$$
\begin{aligned}
& \left\|T\left(I-e^{-t^{2} L}\right)^{M} F_{\beta, M, r}(L) L^{M-\beta} b\right\|_{L^{2}\left(U_{j}\left(B^{t}\right)\right)} \\
& \quad \leq \sum_{i=1}^{\infty}\left\|T\left(I-e^{-t^{2} L}\right)^{M} \chi_{U_{i}\left(B^{t}\right)} F_{\beta, M, r}(L) L^{M-\beta} b\right\|_{L^{2}\left(U_{j}\left(B^{t}\right)\right)} .
\end{aligned}
$$

For $|i-j| \leq 4$, by $L^{2}$ boundedness of $T\left(I-e^{-t^{2} L}\right)^{M}$ and off-diagonal estimates (3.6),

$$
\begin{aligned}
& \left\|T\left(I-e^{-t^{2} L}\right)^{M} \chi_{U_{i}\left(B^{t}\right)} F_{\beta, M, r}(L) L^{M-\beta} b\right\|_{L^{2}\left(U_{j}\left(B^{t}\right)\right)} \\
& \quad \leq\left\|F_{\beta, M, r}(L) L^{M-\beta} b\right\|_{L^{2}\left(U_{i}\left(B^{t}\right)\right)} \\
& \quad \leq C e^{-c 4^{i}}\left\|L^{M-\beta} b\right\|_{L^{2}} .
\end{aligned}
$$

For $i \leq j-4$, we decompose $U_{i}\left(B^{t}\right)$ as the union of a finite number of balls $B_{\kappa, i}=B\left(x_{\kappa, i}, t\right)$, the number is compared with $2^{i D}$ and $\operatorname{dist}\left(B_{\kappa, i}, B\right) \geq C 2^{i} r$. For each $B_{\kappa, i}$, we can write

$$
U_{j}\left(B^{t}\right) \subset \bigcup_{\ell=0}^{2 i} U_{j-i+\ell}\left(B_{\kappa, i}\right)
$$

Thus by condition (3.1) and off-diagonal estimates (3.6),

$$
\begin{aligned}
& \left\|T\left(I-e^{-t^{2} L}\right)^{M} \chi_{U_{i}\left(B^{t}\right)} F_{\beta, M, r}(L) L^{M-\beta} b\right\|_{L^{2}\left(U_{j}\left(B^{t}\right)\right)} \\
& \quad \leq \sum_{\kappa} \sum_{\ell}\left\|T\left(I-e^{-t^{2} L}\right)^{M} \chi_{B_{\kappa, i}} F_{\beta, M, r}(L) L^{M-\beta} b\right\|_{L^{2}\left(U_{j-i+\ell}\left(B_{\kappa, i}\right)\right)}
\end{aligned}
$$




$$
\begin{aligned}
& \leq C \sum_{\kappa} \sum_{\ell} 2^{-(j-i+\ell) s}\left\|F_{\beta, M, r}(L) L^{M-\beta} b\right\|_{L^{2}\left(B_{\kappa, i}\right)} \\
& \leq C \sum_{\kappa} \sum_{\ell} 2^{-(j-i+\ell) s} e^{-c 4^{i}}\left\|L^{M-\beta} b\right\|_{L^{2}} \\
& \leq C 2^{i D} 2^{-(j-i) s} e^{-c 4^{i}}\left\|L^{M-\beta} b\right\|_{L^{2}} .
\end{aligned}
$$

Thus

$$
\begin{aligned}
& \sum_{i=1}^{j-4}\left\|T\left(I-e^{-t^{2} L}\right)^{M} \chi_{U_{i}\left(B^{t}\right)} F_{\beta, M, r}(L) L^{M-\beta} b\right\|_{L^{2}\left(U_{j}\left(B^{t}\right)\right)} \\
& \quad \leq C 2^{-j s}\left\|L^{M-\beta} b\right\|_{L^{2}} \sum_{i=1}^{j-4} 2^{i D+i s} e^{-c 4^{i}} \leq C 2^{-j s}\left\|L^{M-\beta} b\right\|_{L^{2}}
\end{aligned}
$$

For $i \geq j+4$, decompose $U_{i}\left(B^{t}\right)$ as the union of finite number of balls $B_{\kappa, i}=B\left(x_{\kappa, i}, t\right)$, the number is compared with $2^{i D}$ and $\operatorname{dist}\left(B_{\kappa, i}, B\right) \geq C 2^{i} r$. For any $B_{\kappa, i}$, we can write

$$
U_{j}\left(B^{t}\right) \subset \bigcup_{\ell=-2}^{2 j+1} U_{i-j+\ell}\left(B_{\kappa, i}\right) .
$$

Thus by condition (3.1) and off-diagonal estimates (3.6),

$$
\begin{aligned}
& \left\|T\left(I-e^{-t^{2} L}\right)^{M} \chi_{U_{i}\left(B^{t}\right)} F_{\beta, M, r}(L) L^{M-\beta} b\right\|_{L^{2}\left(U_{j}\left(B^{t}\right)\right)} \\
& \leq \sum_{\kappa} \sum_{\ell}\left\|T\left(I-e^{-t^{2} L}\right)^{M} \chi_{B_{\kappa, i}} F_{\beta, M, r}(L) L^{M-\beta} b\right\|_{L^{2}\left(U_{i-j+\ell}\left(B_{\kappa, i}\right)\right)} \\
& \leq C \sum_{\kappa} \sum_{\ell} 2^{-(i-j+\ell) s}\left\|F_{\beta, M, r}(L) L^{M-\beta} b\right\|_{L^{2}\left(B_{\kappa, i}\right)} \\
& \leq C \sum_{\kappa} \sum_{\ell} 2^{-(i-j+\ell) s} e^{-c 4^{i}}\left\|L^{M-\beta} b\right\|_{L^{2}} \\
& \quad \leq C 2^{i D} 2^{-(i-j) s} e^{-c 4^{i}}\left\|L^{M-\beta} b\right\|_{L^{2}} .
\end{aligned}
$$

Thus

$$
\begin{aligned}
& \sum_{i=j+4}^{\infty}\left\|T\left(I-e^{-t^{2} L}\right)^{M} \chi_{U_{i}\left(B^{t}\right)} F_{\beta, M, r}(L) L^{M-\beta} b\right\|_{L^{2}\left(U_{j}\left(B^{t}\right)\right)} \\
& \leq C 2^{j s}\left\|L^{M-\beta} b\right\|_{L^{2}} \sum_{i=j+4}^{\infty} 2^{i D-i s} e^{-c 4^{i}} \leq C 2^{-j s}\left\|L^{M-\beta} b\right\|_{L^{2}}
\end{aligned}
$$

Combining the estimates (3.8), (3.9), (3.10) and (3.11), it follows that

$$
\left\|T\left(I-e^{-t^{2} L}\right)^{M} F_{\beta, M, r}(L) L^{M-\beta} b\right\|_{L^{2}\left(U_{j}\left(B^{t}\right)\right)} \leq C 2^{-j s}\left\|L^{M-\beta} b\right\|_{L^{2}} .
$$

Noting that for $t \in[r, \sqrt{2} r]$, we have

$$
U_{j}(B) \subset U_{j}\left(B^{t}\right) \cup U_{j-1}\left(B^{t}\right) .
$$


Thus, by (3.7) and by (iii) of Defintion 2.6,

$$
\begin{aligned}
\|T a\|_{L^{1}\left(U_{j}(B)\right)} & \leq C v\left(U_{j}(B)\right)^{\frac{1}{2}} r^{-2 \beta} 2^{-j s}\left\|L^{M-\beta} b\right\|_{L^{2}} \\
& \leq C 2^{-j s} v\left(U_{j}(B)\right)^{\frac{1}{2}} r^{-2 \beta} r^{2 \beta} v(B)^{-\frac{1}{2}} \\
& \leq C 2^{-j(s-D / 2)}
\end{aligned}
$$

which proves (3.4). Then combining estimate (3.3), we complete the proof of Lemma 3.2.

Now we state the main result of this section.

Theorem 3.3. Let $M$ be a complete non-compact Riemannian manifold satisfying assumptions (1.1) with doubling dimension D, Gaussian upper bound (1.2) and $\alpha$-subcritical condition (1.3). Then the associated Riesz transform $d^{*} \vec{\Delta}^{-\frac{1}{2}}$ is

i) bounded from $H_{\vec{\Delta}}^{1}\left(\Lambda^{1} T^{*} M\right)$ to $L^{1}(M)$,

ii) bounded from $H_{\vec{\Delta}}^{p}\left(\Lambda^{1} T^{*} M\right)$ to $L^{p}(M)$ for all $p \in[1,2]$,

iii) bounded from $L^{p}\left(\Lambda^{1} T^{*} M\right)$ to $L^{p}(M)$ for all $p \in(1,2]$ if $D \leq 2$ and all $p \in\left(p_{0}^{\prime}, 2\right]$ if $D>2$ where $p_{0}:=\frac{2 D}{(D-2)(1-\sqrt{1-\alpha})}$.

Proof. We apply Lemma 3.2. Let $X=M, T X=\Lambda^{1} T^{*} M$ and $L=\vec{\Delta}$.

The estimate (3.1) was proved in the proof of Theorem 1.1 in [24] (the proof of estimate (34) in Page 23). This gives assertion i).

Assertion ii) follows from i) by interpolation and the fact that $d^{*} \vec{\Delta}^{-\frac{1}{2}}$ is bounded from $L^{2}\left(\Lambda^{1} T^{*} M\right)$ to $L^{2}(M)$.

Finally, the Davies-Gaffney estimate $\left(D G_{p}\right)$ was proved in [24], Theorem 4.1. We then apply Proposition 2.5 to obtain iii).

By duality and the commutation formula

$$
\vec{\Delta} d=d \Delta
$$

we obtain the following corollary of Theorem 3.3.

Corollary 3.4. Let $M$ be a complete non-compact Riemannian manifold satisfying assumptions (1.1) with doubling dimension D, Gaussian upper bound (1.2) and $\alpha$-subcritical condition (1.3). Then the associated Riesz transform $d \Delta^{-\frac{1}{2}}$ is

i) bounded from $L^{p}(M)$ to $H_{\vec{\Delta}}^{p}\left(\Lambda^{1} T^{*} M\right)$ for all $p \in[2, \infty)$,

ii) bounded from $L^{p}(M)$ to $L^{p}\left(\Lambda^{1} T^{*} M\right)$ for all $p \in[2, \infty)$ if $D \leq 2$ and all $p \in\left[2, p_{0}\right)$ if $D>2$ where $p_{0}:=\frac{2 D}{(D-2)(1-\sqrt{1-\alpha})}$.

As mentioned in the introduction, assertion ii) was already proved in [24].

\section{The RIESz TRANSForm FOR $p>2$}

Our aim in this section is to investigate boundedness of the Riesz transform $d \Delta^{-\frac{1}{2}}$ on $L^{p}$ for other values of $p>2$ that are not covered by the previous corollary. In order to do this we make an integrability assumption on the Ricci curvature.

Our main result in this section is the following theorem. 
Theorem 4.1. Assume that the Riemannian manifold $M$ satisfies the doubling condition (1.1) and the Gaussian upper bound (1.2). Assume that the negative part $R^{-}$of the Ricci curvature $R$ satisfies (1.4) for some $p_{1}$ and $p_{2}$ such that $3<p_{2} \leq D$. Then the Riesz transform $d \Delta^{-\frac{1}{2}}$ is bounded from $L^{p}(M)$ to $L^{p}\left(\Lambda^{1} T^{*} M\right)$ for $1<p<p_{2}$.

Remark 4.2. Suppose that $v(x, r) \geq C v(r)$ for all $r>0$. Then (1.4) is satisfied for $p_{1}$ and $p_{2}$ such that

$$
\int_{0}^{1} v(t)^{-1 / p_{1}} d t<\infty \text { and } R^{-} \in L^{\frac{p_{1}}{2}}
$$

and

$$
\int_{1}^{\infty} v(t)^{-1 / p_{2}} d t<\infty \text { and } R^{-} \in L^{\frac{p_{2}}{2}}
$$

In the particular case where $v(r)=r^{D}$ for all $r>0,(1.4)$ is then satisfied if $R^{-} \in L^{\frac{D}{2}-\eta} \cap L^{\frac{D}{2}+\eta}$ for some $\eta>0$. Therefore, the Riesz transform $d \Delta^{-\frac{1}{2}}$ is bounded from $L^{p}(M)$ to $L^{p}\left(\Lambda^{1} T^{*} M\right)$ for all $p$ with $1<p<D$. We recover and extend a result of Devyver [14] who assumed $R^{-} \in L^{\frac{D}{2}-\eta} \cap L^{\infty}$.

Before we start the proof of the theorem we state the following result on $L^{p}-L^{q}$ estimates for perturbations of $\vec{\Delta}$ by a non-negative potential. The manifold $M$ satisfies the same assumptions as in the previous theorem.

Theorem 4.3. Let $\mathcal{R}=\mathcal{R}^{+}-\mathcal{R}^{-}$be a field of symmetric endomorphisms acting on $\Lambda^{1} T^{*} M$. Let $\alpha \in[0,1)$. We suppose that $\mathcal{R}^{-}$is $\alpha$-subcritical for the operator $\nabla^{*} \nabla+\mathcal{R}^{+}$, that is for all $\omega \in \mathcal{C}_{c}^{\infty}\left(\Lambda^{1} T^{*} M\right)$

$$
\left(\mathcal{R}^{-} \omega, \omega\right) \leq \alpha\left(\left(\nabla^{*} \nabla+\mathcal{R}^{+}\right) \omega, \omega\right)
$$

Then for every open subsets $E$ and $F$ of $M$

i) $\left\|\chi_{E} d^{*} e^{-t\left(\nabla^{*} \nabla+\mathcal{R}^{+}-\mathcal{R}^{-}\right)} \chi_{F}\right\|_{2 \rightarrow 2} \leq \frac{C}{\sqrt{t}} e^{-c \frac{\operatorname{dist}(E, F)^{2}}{t}}$

ii) $\left\|e^{-t\left(\nabla^{*} \nabla+\mathcal{R}^{+}-\mathcal{R}^{-}\right)} \chi_{B(x, r)}\right\|_{p \rightarrow q} \leq \frac{C}{v(x, r)^{\frac{1}{p}-\frac{1}{q}}} \max \left(\frac{r}{\sqrt{t}}, \frac{\sqrt{t}}{r}\right)^{\beta}$,

where $C, c$ and $\beta$ are positive constants. The assertion ii) holds for all $p \leq q \in(1, \infty)$ if $D \leq 2$ and all $p \in\left(p_{0}^{\prime}, p_{0}\right), q \in\left[p, p_{0}\right)$ if $D>2$ where $p_{0}:=\frac{2 D}{(D-2)(1-\sqrt{1-\alpha})}$.

If $\mathcal{R}$ is the Ricci curvature, this theorem was proved in [24], Theorem 4.1 and Corollary 4.5. In the general case, the proof is the same as in [24].

Proof of Theorem 4.1. We shall proceed in three main steps.

Step I. For a fixed point $x_{0} \in M$, we prove that there exist a positive number $r_{0}$ sufficiently large and a positive function $W \in C_{c}^{\infty}(M)$ with supp $W \subset B\left(x_{0}, r_{0}\right)$ such that the following $L^{p}-L^{q}$ estimates hold for all $p \in\left(p_{0}^{\prime}, p_{0}\right), q \in\left[p, p_{0}\right)$ and $t>1$

$$
\left\|e^{-t(\vec{\Delta}+W)} \chi_{B\left(x_{0}, r_{0}\right)}\right\|_{p \rightarrow q} \leq C_{x_{0}, r_{0}} t^{-p_{2}\left(\frac{1}{p}-\frac{1}{q}\right) / 2}
$$

For any $\varepsilon>0$, by the Dominated Convergence Theorem, we can find a large enough $r_{0}$ such that

$$
\int_{0}^{1}\left\|\frac{\left|R^{-}\right|^{\frac{1}{2}}}{v(\cdot, \sqrt{t})^{\frac{1}{p_{1}}}}\right\|_{L^{p_{1}\left(B\left(x_{0}, \frac{r_{0}}{2}\right)^{c}\right)}} \frac{d t}{\sqrt{t}}+\int_{1}^{\infty}\left\|\frac{\left|R^{-}\right|^{\frac{1}{2}}}{v(\cdot, \sqrt{t})^{\frac{1}{p_{2}}}}\right\|_{L^{p_{2}\left(B\left(x_{0}, \frac{r_{0}}{2}\right)^{c}\right)}} \frac{d t}{\sqrt{t}}<\varepsilon .
$$


We construct a function $0 \leq \varphi \in C_{c}^{\infty}(M)$ such that $\varphi=1$ in the ball $B\left(x_{0}, r_{0} / 2\right), \varphi \leq 1$ in $B\left(x_{0}, r_{0}\right) \backslash B\left(x_{0}, r_{0} / 2\right)$ and $\varphi=0$ outside the ball $B\left(x_{0}, r_{0}\right)$. Let $V=\varphi\left|R^{-}\right|$. Then $V$ is a compactly supported function and smooth except when $\left|R^{-}\right|=0$. We choose another function $W$ such that $W \geq V, W \in C_{c}^{\infty}(M)$ and $\operatorname{supp} W \subset B\left(x_{0}, r_{0}\right)$. We write

$$
\vec{\Delta}+W=\nabla^{*} \nabla+R^{+}+\left(W-R^{-}\right)^{+}-\left(W-R^{-}\right)^{-} .
$$

Note that $\left(W-R^{-}\right)^{-}=\left(W-V+V-R^{-}\right)^{-} \leq\left(V-R^{-}\right)^{-}$and $\left|\left(V-R^{-}\right)^{-}\right|=0$ in the ball $B\left(x_{0}, r_{0} / 2\right)$ and $\left|\left(V-R^{-}\right)^{-}\right| \leq\left|R^{-}\right|$outside the ball $B\left(x_{0}, r_{0} / 2\right)$. Therefore, $\left\|\left(W-R^{-}\right)^{-}\right\|_{\text {vol }}<$ $\varepsilon$. From Proposition 5.8 in [24], with $\left(W-R^{-}\right)^{-}$and $\nabla^{*} \nabla+R^{+}+\left(W-R^{-}\right)^{+}$instead of $R^{-}$ and $\nabla^{*} \nabla+R^{+}$, we have

$$
\left(\left(W-R^{-}\right)^{-} \omega, \omega\right) \leq C\left\|\left(W-R^{-}\right)^{-}\right\|_{v o l}^{2}\left(\left(\nabla^{*} \nabla+R^{+}+\left(W-R^{-}\right)^{+}\right) \omega, \omega\right) .
$$

Then we choose $\varepsilon$ small enough to have $C\left\|\left(W-R^{-}\right)^{-}\right\|_{v o l}<C \varepsilon<1$. Therefore, $\left(W-R^{-}\right)^{-}$ is $\varepsilon$-subcritical with respect to $\nabla^{*} \nabla+R^{+}+\left(W-R^{-}\right)^{+}$, i.e.

$$
\left(\left(W-R^{-}\right)^{-} \omega, \omega\right) \leq \varepsilon\left(\left(\nabla^{*} \nabla+R^{+}+\left(W-R^{-}\right)^{+}\right) \omega, \omega\right) \text { for all } \omega \in C_{c}^{\infty}\left(\Lambda^{1} T^{*} M\right) .
$$

By Theorem 4.3, we obtain $L^{p}-L^{q}$ off-diagonal estimates for $e^{-t(\vec{\Delta}+W)}$ for all $p \in\left(p_{0}^{\prime}, p_{0}\right), q \in$ $\left[p, p_{0}\right)$ with $p_{0}:=\frac{2 D}{(D-2)(1-\sqrt{1-\varepsilon})}$. Hence for $p \in\left(p_{0}^{\prime}, p_{0}\right), q \in\left[p, p_{0}\right)$ and $\sqrt{t} \geq r_{0}$

$$
\left\|e^{-t(\vec{\Delta}+W)} \chi_{B\left(x_{0}, r_{0}\right)}\right\|_{p \rightarrow q} \leq\left\|e^{-t(\vec{\Delta}+W)} \chi_{B\left(x_{0}, \sqrt{t}\right)}\right\|_{p \rightarrow q} \leq C v\left(x_{0}, \sqrt{t}\right)^{-\left(\frac{1}{p}-\frac{1}{q}\right)},
$$

and for $1<\sqrt{t} \leq r_{0}$

$$
\left\|e^{-t(\vec{\Delta}+W)} \chi_{B\left(x_{0}, r_{0}\right)}\right\|_{p \rightarrow q} \leq \frac{C}{v\left(x_{0}, r_{0}\right)^{\frac{1}{p}-\frac{1}{q}}}\left(\frac{r_{0}}{\sqrt{t}}\right)^{\beta} \leq C_{x_{0}, r_{0}} t^{-p_{2}\left(\frac{1}{p}-\frac{1}{q}\right) / 2} .
$$

Note that $|V| \leq\left|R^{-}\right|$and so $\|V\|_{\text {vol }} \leq\left\|R^{-}\right\|_{\text {vol }} \leq C$, which gives

$$
\int_{1}^{\infty}\left\|\frac{V^{\frac{1}{2}}}{v(\cdot, \sqrt{t})^{\frac{1}{p_{2}}}}\right\|_{p_{2}} \frac{d t}{\sqrt{t}} \leq C
$$

Since $\operatorname{supp} V \subset B\left(x_{0}, r_{0}\right)$, we have for $x \in B\left(x_{0}, r_{0}\right)$ and $\sqrt{t}>r_{0}$

$$
v(x, \sqrt{t})=\frac{v(x, \sqrt{t})}{v\left(x_{0}, \sqrt{t}\right)} v\left(x_{0}, \sqrt{t}\right) \leq C\left(1+\frac{\rho\left(x, x_{0}\right)}{\sqrt{t}}\right)^{D} v\left(x_{0}, \sqrt{t}\right) \leq C^{\prime} v\left(x_{0}, \sqrt{t}\right) .
$$

Thus

$$
\left\|V^{\frac{1}{2}}\right\|_{p_{2}} \int_{r_{0}^{2}}^{\infty} \frac{1}{v\left(x_{0}, \sqrt{t}\right)^{\frac{1}{p_{2}}}} \frac{d t}{\sqrt{t}} \leq C^{\prime} \int_{r_{0}^{2}}^{\infty}\left\|\frac{V^{\frac{1}{2}}}{v(\cdot, \sqrt{t})^{\frac{1}{p_{2}}}}\right\|_{p_{2}} \frac{d t}{\sqrt{t}} \leq C .
$$

Note that if $V=0$, then $R^{-}=0$ in the ball $B\left(x_{0}, r_{0} / 2\right)$ and so $\left\|R^{-}\right\|_{v o l}<\varepsilon$. As a consequence, $R^{-}$satisfies the $\varepsilon$-subcritical condition (1.3). By Corollary 1.2 in [24] or our Corollary 3.4, $d \Delta^{-\frac{1}{2}}$ is bounded on $L^{p}$ for $p \in\left(1, p_{0}\right)$ where $p_{0}:=\frac{2 D}{(D-2)(1-\sqrt{1-\varepsilon})}$. With our choice of $\varepsilon$, we have 
$d \Delta^{-\frac{1}{2}}$ bounded on $L^{p}$ for $p \in\left(1, p_{2}\right)^{1}$. In the sequel, we assume that $V \neq 0$. Hence

$$
\int_{r_{0}}^{\infty} \frac{1}{v\left(x_{0}, s\right)^{\frac{1}{p_{2}}}} d s \leq \int_{r_{0}^{2}}^{\infty} \frac{1}{v\left(x_{0}, \sqrt{t}\right)^{\frac{1}{p_{2}}}} \frac{d t}{\sqrt{t}} \leq C\left\|V^{\frac{1}{2}}\right\|_{p_{2}}^{-1} \leq C^{\prime} .
$$

Let $f(s)=v\left(x_{0}, s\right)^{-\frac{1}{p_{2}}}$. Note that $f$ is a positive continuous decreasing function. So by the first mean value theorem, there exists $\xi \in\left[r_{0}, t\right]$ such that

$$
\int_{r_{0}}^{t} f(s) d s=f(\xi)\left(t-r_{0}\right) \geq f(t) t-f\left(r_{0}\right) r_{0} .
$$

We deduce that for $t>r_{0}$

$$
0<t f(t) \leq r_{0} f\left(r_{0}\right)+\int_{r_{0}}^{t} f(s) d s \leq r_{0} f\left(r_{0}\right)+\int_{r_{0}}^{\infty} f(s) d s \leq C .
$$

That is for $t>r_{0}$

$$
f(t) \leq C / t
$$

and

$$
v\left(x_{0}, t\right) \geq C t^{p_{2}} .
$$

Therefore by $(4.2),(4.3)$ and $(4.4)$, we have the following estimate for $p \in\left(p_{0}^{\prime}, p_{0}\right), q \in\left[p, p_{0}\right)$ and all $t>1$

$$
\left\|e^{-t(\vec{\Delta}+W)} \chi_{B\left(x_{0}, r_{0}\right)}\right\|_{p \rightarrow q} \leq C_{x_{0}, r_{0}} t^{-p_{2}\left(\frac{1}{p}-\frac{1}{q}\right) / 2} .
$$

Step II. We prove that the operator $d(\Delta+W)^{-\frac{1}{2}}$ is bounded on $L^{p}$ for all $p \in\left[2, p_{2}\right)$. In order to do this we take the difference with $(\vec{\Delta}+W)^{-\frac{1}{2}} d$, that is,

$$
d(\Delta+W)^{-\frac{1}{2}}=d(\Delta+W)^{-\frac{1}{2}}-(\vec{\Delta}+W)^{-\frac{1}{2}} d+(\vec{\Delta}+W)^{-\frac{1}{2}} d .
$$

It follows from (4.1) and [24, Theorem 1.1] or our Theorem 3.3 that $d^{*}(\vec{\Delta}+W)^{-\frac{1}{2}}$ is bounded on $L^{p}\left(\Lambda^{1} T^{*} M\right)$ to $L^{p}(M)$ for all $p \in\left(p_{0}^{\prime}, 2\right]$ with again $p_{0}:=\frac{2 D}{(D-2)(1-\sqrt{1-\varepsilon})}$. By duality, $(\vec{\Delta}+W)^{-\frac{1}{2}} d$ is bounded from $L^{p}(M)$ to $L^{p}\left(\Lambda^{1} T^{*} M\right)$ for all $p \in\left[2, p_{0}\right)$. Choosing again $\varepsilon$ small enough such that $p_{0} \geq p_{2}$, it follows that $(\vec{\Delta}+W)^{-\frac{1}{2}} d$ is bounded on $L^{p}(M)$ to $L^{p}\left(\Lambda^{1} T^{*} M\right)$ for all $p \in\left[2, p_{2}\right)$. It remains to prove the boundedness of $d(\Delta+W)^{-\frac{1}{2}}-(\vec{\Delta}+W)^{-\frac{1}{2}} d$. For this part we follow the strategy in [14, Section 5.2] and [9, Section 3.2]. In these two papers, the authors assume a global Sobolev inequality on the manifold together with a polynomial lower bound on the volume. We adapt their ideas to our setting.

Let $\square_{t}=-\frac{\partial^{2}}{\partial t^{2}}+(\vec{\Delta}+W)$. We have for $u \in C_{c}^{\infty}(M)$

$$
\square_{t}\left(d e^{-t \sqrt{\Delta+W}} u-e^{-t \sqrt{\vec{\Delta}+W}} d u\right)=\square_{t} d e^{-t \sqrt{\Delta+W}} u=-\left(e^{-t \sqrt{\Delta+W}} u\right) d W .
$$

The operator $L_{1}:=-\frac{\partial^{2}}{\partial t^{2}}$ with domain

$$
D\left(L_{1}\right)=W^{2,2}\left((0, \infty), L^{2}\left(\Lambda^{1} T^{*} M\right)\right) \cap W_{0}^{1,2}\left((0, \infty), L^{2}\left(\Lambda^{1} T^{*} M\right)\right)
$$

\footnotetext{
${ }^{1}$ if $R^{-}=0$, then the Ricci curvature is non-negative and it is well known that the Riesz transform is bounded on $L^{p}$ for all $p \in(1, \infty)$
} 
is self-adjoint on $L^{2}\left((0, \infty), L^{2}\left(\Lambda^{1} T^{*} M\right)\right)$. We define $L_{2}$ as an "extension" of $\vec{\Delta}+W$ to $L^{2}\left((0, \infty), L^{2}\left(\Lambda^{1} T^{*} M\right)\right)$ in the following usual way

$$
\left(L_{2} w\right)(t, x):=(\vec{\Delta}+W)(w(t, \cdot))(x)
$$

with domain

$$
D\left(L_{2}\right):=\left\{w \in L^{2}\left((0, \infty), L^{2}\left(\Lambda^{1} T^{*} M\right)\right): w(t, x) \in D(\vec{\Delta}+W) \text { for a.e. } \mathrm{t}\right\} .
$$

The operators $L_{1}$ and $L_{2}$ are self-adjoint and commute. Therefore, $e^{-s L_{1}} e^{-s L_{2}}=e^{-s L_{2}} e^{-s L_{1}}$ is a strongly continuous semigroup whose generator $\mathcal{C}$ is the closure of $L_{1}+L_{2}$ on the domain $D\left(L_{1}\right) \cap D\left(L_{2}\right)$ (see for example [17], p. 64).

Let $\phi=d e^{-t \sqrt{\Delta+W}} u-e^{-t \sqrt{\vec{\Delta}+W}} d u$. Assume for a moment that $\phi \in D\left(L_{1}\right) \cap D\left(L_{2}\right)$ and that $\mathcal{C}$ is injective. Then

$$
\mathcal{C} \phi=\square_{t} \phi=-\left(e^{-t \sqrt{\Delta+W}} u\right) d W
$$

and we have

$$
\begin{aligned}
d e^{-t \sqrt{\Delta+W}} u-e^{-t \sqrt{\vec{\Delta}+W}} d u & =\phi=-\mathcal{C}^{-1}\left(\left(e^{-t \sqrt{\Delta+W}} u\right) d W\right) \\
& =-\int_{0}^{\infty} e^{-s \mathcal{C}}\left(\left(e^{-t \sqrt{\Delta+W}} u\right) d W\right) d s \\
& =-\int_{0}^{\infty} e^{-s L_{1}} e^{-s L_{2}}\left(\left(e^{-t \sqrt{\Delta+W}} u\right) d W\right) d s \\
& =\int_{0}^{\infty} \int_{0}^{\infty} K_{s}(t, \sigma) e^{-s(\vec{\Delta}+W)}\left(\left(e^{-t \sqrt{\Delta+W}} u\right) d W\right) d \sigma d s
\end{aligned}
$$

where

$$
K_{s}(t, \sigma)=\frac{e^{-\frac{(\sigma+t)^{2}}{4 s}}-e^{-\frac{(\sigma-t)^{2}}{4 s}}}{\sqrt{4 \pi s}}
$$

is the heat kernel on the half-line $\mathbb{R}_{+}$for the Dirichlet boundary condition at 0 .

Next we write

$$
\begin{aligned}
d(\Delta & +W)^{-\frac{1}{2}} u-(\vec{\Delta}+W)^{-\frac{1}{2}} d u \\
& =\int_{0}^{\infty}\left(d e^{-t \sqrt{\Delta+W}} u-e^{-t \sqrt{\vec{\Delta}+W}} d u\right) d t \\
& =\int_{0}^{\infty} \int_{0}^{\infty} \int_{0}^{\infty} K_{s}(t, \sigma) e^{-s(\vec{\Delta}+W)}\left(\left(e^{-t \sqrt{\Delta+W}} u\right) d W\right) d \sigma d s d t=: G(u) .
\end{aligned}
$$

Because $W \geq 0$ and $e^{-t \Delta}$ satisfies the Gaussian upper bound (1.2), it follows that $e^{-t(\Delta+W)}$ also satisfies the same bound (this follows from the domination property $\left|e^{-t(\Delta+W)} f\right| \leq e^{-t \Delta}|f|$ ). Therefore,

$$
\left\|e^{-t(\Delta+W)} \chi_{B\left(x_{0}, r_{0}\right)}\right\|_{p \rightarrow 2} \leq C v\left(x_{0}, \sqrt{t}\right)^{-\left(\frac{1}{p}-\frac{1}{2}\right)}\left(1+\frac{r_{0}}{\sqrt{t}}\right)^{D}
$$

It follows from the subordination formula $e^{-t \sqrt{A}}=\frac{1}{\sqrt{\pi}} \int_{0}^{\infty} \frac{e^{-u}}{\sqrt{u}} e^{-\frac{t^{2} A}{4 u}} d u$ that

$$
\left\|e^{-t \sqrt{\Delta+W}} \chi_{B\left(x_{0}, r_{0}\right)}\right\|_{p \rightarrow 2} \leq C v\left(x_{0}, t\right)^{-\left(\frac{1}{p}-\frac{1}{2}\right)}\left(1+\frac{r_{0}}{t}\right)^{D}
$$


By the volume condition (4.4) and the doubling condition (1.1), we have for all $p \in[1,2]$ and $t>1$,

$$
\left\|e^{-t \sqrt{\Delta+W}} \chi_{B\left(x_{0}, r_{0}\right)}\right\|_{p \rightarrow 2} \leq C_{x_{0}, r_{0}} t^{-p_{2}\left(\frac{1}{p}-\frac{1}{2}\right)},
$$

and similarly for all $p \geq 2$ and $t \geq 1$

$$
\left\|\chi_{B\left(x_{0}, r_{0}\right)} e^{-t \sqrt{\Delta+W}}\right\|_{p \rightarrow \infty} \leq C_{x_{0}, r_{0}} t^{-\frac{p_{2}}{p}} .
$$

From these estimates we want to obtain that $\|G(u)\|_{p} \leq C\|u\|_{p}$ for all $p \in\left[2, p_{2}\right)$. Since these estimates are valid for $t>1$ we have to treat first the case of small $t$ and $s$ in the definition of $G$.

Let $g_{s, t}(u):=e^{-s(\vec{\Delta}+W)}\left(\left(e^{-t \sqrt{\Delta+W}} u\right) d W\right)$. Then

$$
\begin{aligned}
G(u) & =\int_{0}^{\infty} \int_{0}^{\infty} \int_{0}^{\infty} K_{s}(t, \sigma) g_{s, t}(u) d \sigma d s d t \\
& =\int_{0}^{\infty} \int_{0}^{\infty} \int_{0}^{\infty} \frac{e^{-\frac{(\sigma+t)^{2}}{4 s}}-e^{-\frac{(\sigma-t)^{2}}{4 s}}}{\sqrt{4 \pi s}} g_{s, t}(u) d \sigma d s d t \\
& =\int_{0}^{\infty} \int_{0}^{\infty} \frac{g_{s, t}(u)}{\sqrt{4 \pi s}}\left[\int_{0}^{\infty} e^{-\frac{(\sigma+t)^{2}}{4 s}}-e^{-\frac{(\sigma-t)^{2}}{4 s}} d \sigma\right] d s d t \\
& =-\int_{0}^{\infty} \int_{0}^{\infty} \frac{g_{s, t}(u)}{\sqrt{4 \pi s}}\left[\int_{-t}^{t} e^{-\frac{\sigma^{2}}{4 s}} d \sigma\right] d s d t \\
& =-\int_{0}^{\infty} \int_{0}^{\infty} \frac{2 g_{s, t}(u)}{\sqrt{\pi}}\left[\int_{0}^{\frac{t}{2 \sqrt{s}}} e^{-\gamma^{2}} d \gamma\right] d s d t \\
& =-\frac{2}{\sqrt{\pi}} \int_{0}^{\infty} \int_{0}^{\infty} e^{-\gamma^{2}}\left[\int_{0}^{\frac{t^{2}}{4 \gamma^{2}}} g_{s, t}(u) d s\right] d \gamma d t .
\end{aligned}
$$

Thus

$$
\|G(u)\|_{L^{p}} \leq \frac{2}{\sqrt{\pi}} \int_{0}^{\infty} \int_{0}^{\infty} e^{-\gamma^{2}}\left[\int_{0}^{\frac{t^{2}}{4 \gamma^{2}}}\left\|g_{s, t}(u)\right\|_{L^{p}} d s\right] d \gamma d t .
$$

For all $s \in[0,1]$ and $t \in[0,1]$, by the fact that the semigroup $e^{-s(\vec{\Delta}+W)}$ and $e^{-t \sqrt{\Delta+W}}$ are uniformly bounded on $L^{p}$ for all $s>0$ and $t>0$,

$$
\left\|g_{s, t}(u)\right\|_{L^{p}}=\left\|e^{-s(\vec{\Delta}+W)}\left(\left(e^{-t \sqrt{\Delta+W}} u\right) d W\right)\right\|_{L^{p}} \leq C\|d W\|_{L^{\infty}}\|u\|_{L^{p}} .
$$

For all $s \in[0,1]$ and $t>1$, by the fact that the semigroup $e^{-s(\vec{\Delta}+W)}$ is uniformly bounded on $L^{p}$ for all $s>0$ and estimate (4.7),

$$
\begin{aligned}
\left\|g_{s, t}(u)\right\|_{L^{p}} & =\left\|e^{-s(\vec{\Delta}+W)}\left(\left(e^{-t \sqrt{\Delta+W}} u\right) d W\right)\right\|_{L^{p}} \\
& \leq C\left\|\left(e^{-t \sqrt{\Delta+W}} u\right) d W\right\|_{L^{p}} \\
& \leq C\|d W\|_{L^{p}}\left\|\chi_{B\left(x_{0}, r_{0}\right)} e^{-t \sqrt{\Delta+W}} u\right\|_{L^{\infty}} \\
& \leq C C_{x_{0}, r_{0}}\|d W\|_{L^{p}} t^{-\frac{p_{2}}{p}}\|u\|_{L^{p}} .
\end{aligned}
$$


For all $s>1$ and $t \in[0,1]$, by the fact that the semigroup $e^{-t \sqrt{\Delta+W}}$ is uniformly bounded on $L^{p}$ for all $t>0$ and estimate (4.5),

$$
\begin{aligned}
\left\|g_{s, t}(u)\right\|_{L^{p}} & =\left\|e^{-s(\vec{\Delta}+W)}\left(\left(e^{-t \sqrt{\Delta+W}} u\right) d W\right)\right\|_{L^{p}} \\
& \leq C\left\|e^{-s(\vec{\Delta}+W)} \chi_{B\left(x_{0}, r_{0}\right)}\right\|_{\left(p_{0}^{\prime}+\varepsilon\right) \rightarrow p}\left\|\left(e^{-t \sqrt{\Delta+W}} u\right) d W\right\|_{L^{p_{0}^{\prime}+\varepsilon}} \\
& \leq C C_{x_{0}, r_{0}} s^{-p_{2}\left(\frac{1}{p_{0}^{\prime}+\varepsilon}-\frac{1}{p}\right) / 2}\|d W\|_{L}^{1 /\left(\frac{1}{p_{0}^{\prime}+\varepsilon}-\frac{1}{p}\right)}\left\|e^{-t \sqrt{\Delta+W}} u\right\|_{L^{p}} \\
& \leq C C_{x_{0}, r_{0}}\|d W\|_{L}^{1 /\left(\frac{1}{p_{0}^{\prime}+\varepsilon}-\frac{1}{p}\right)} s^{-p_{2}\left(\frac{1}{p_{0}^{\prime}+\varepsilon}-\frac{1}{p}\right) / 2}\|u\|_{L^{p}} .
\end{aligned}
$$

Similarly, for all $s>1$ and $t>1$,

$$
\begin{aligned}
\left\|g_{s, t}(u)\right\|_{L^{p}} & =\left\|e^{-s(\vec{\Delta}+W)}\left(\left(e^{-t \sqrt{\Delta+W}} u\right) d W\right)\right\|_{L^{p}} \\
& \leq C\left\|e^{-s(\vec{\Delta}+W)} \chi_{B\left(x_{0}, r_{0}\right)}\right\|_{\left(p_{0}^{\prime}+\varepsilon\right) \rightarrow p}\left\|\left(e^{-t \sqrt{\Delta+W}} u\right) d W\right\|_{L^{p_{0}^{\prime}+\varepsilon}} \\
& \leq C C_{x_{0}, r_{0}} s^{-p_{2}\left(\frac{1}{p_{0}^{\prime}+\varepsilon}-\frac{1}{p}\right) / 2}\|d W\|_{L}^{1 /\left(\frac{1}{p_{0}^{\prime}+\varepsilon}-\frac{1}{p}\right)}\left\|\chi_{B\left(x_{0}, r_{0}\right)} e^{-t \sqrt{\Delta+W}} u\right\|_{L^{p}} \\
& \leq C C_{x_{0}, r_{0}}\|d W\|_{L}{ }_{L}^{1 /\left(\frac{1}{p_{0}^{\prime}+\varepsilon}-\frac{1}{p}\right)} s^{-p_{2}\left(\frac{1}{p_{0}^{\prime}+\varepsilon}-\frac{1}{p}\right) / 2}\left\|\chi_{B\left(x_{0}, r_{0}\right)}\right\|_{L^{p}}\left\|\chi_{B\left(x_{0}, r_{0}\right)} e^{-t \sqrt{\Delta+W}} u\right\|_{L^{\infty}} \\
& \leq C C_{x_{0}, r_{0}}\|d W\|_{L}^{1 /\left(\frac{1}{p_{0}^{\prime}+\varepsilon}-\frac{1}{p}\right)} s^{-p_{2}\left(\frac{1}{p_{0}^{\prime}+\varepsilon}-\frac{1}{p}\right) / 2}\left\|\chi_{B\left(x_{0}, r_{0}\right)}\right\|_{L^{p}} t^{-\frac{p_{2}}{p}}\|u\|_{L^{p}} .
\end{aligned}
$$

Combing the above four estimates, we get

$$
\left\|g_{s, t}(u)\right\|_{L^{p}} \leq C(1+t)^{-\frac{p_{2}}{p}}(1+s)^{-p_{2}\left(\frac{1}{p_{0}^{\prime}+\varepsilon}-\frac{1}{p}\right) / 2}\|u\|_{L^{p}} .
$$

Putting this estimate into estimate (4.8) and noting that $p>2, p_{2}>3$ and $p<p_{2}$, we have

$$
\|G(u)\|_{L^{p}} \leq C\|u\|_{L^{p}}
$$

Hence, $d(\Delta+W)^{-\frac{1}{2}}$ is bounded on $L^{p}$ for $p \in\left[2, p_{2}\right)$.

Recall that we have used in the previous proof that $\phi \in D\left(L_{1}\right) \cap D\left(L_{2}\right)$ and $\mathcal{C}$ is injective. Now we prove these two properties.

If $\mathcal{C} \psi=0$, then $\psi=e^{-s L_{1}} e^{-s L_{2}} \psi=e^{-s L_{2}} e^{-s L_{1}} \psi$ and the self-adjointness of $L_{1}$ and $L_{2}$ imply that $\psi \in D\left(L_{1}\right) \cap D\left(L_{2}\right)$. Hence

$$
\left\langle\frac{\partial^{2}}{\partial t^{2}} \psi, \psi\right\rangle_{L^{2}\left(\mathbb{R}_{+} \times T M\right)}=0,
$$

which implies $\partial_{t} \psi=0$ and thus $\psi(x, t)=\psi(x)$. In addition, $\psi \in W_{0}^{1,2}$ implies $\psi(x, 0)=0$ and hence $\psi=0$. This shows that $\mathcal{C}$ is injective.

Now we prove that $\phi \in D\left(L_{1}\right) \cap D\left(L_{2}\right)$. For fixed $t$, because $u$ and $W$ belong to $C_{c}^{\infty}$, it is easy to see that $\phi \in L^{2}$ and $(\vec{\Delta}+W) \phi \in L^{2}$. Thus $\phi \in D\left(L_{2}\right)$. Note that

$$
\lim _{t \rightarrow 0} \phi=\lim _{t \rightarrow 0} d e^{-t \sqrt{\Delta+W}} u-\lim _{t \rightarrow 0} e^{-t \sqrt{\vec{\Delta}+W}} d u=d u-d u=0 .
$$

$\left(\lim _{t \rightarrow 0} d e^{-t \sqrt{\Delta+W}} u=d u\right.$ comes from that fact $d(\Delta+W)^{-1 / 2}$ is bounded on $L^{2}$ and $(\Delta+$ $W)^{1 / 2} e^{-t \sqrt{\Delta+W}} u$ converges to $\left.(\Delta+W)^{1 / 2} u\right)$. It remains to check that $\phi \in W^{2,2}\left((0, \infty), L^{2}\left(\Lambda^{1} T^{*} M\right)\right)$. 
We write $\phi=\phi_{1}-\phi_{2}$ where $\phi_{1}:=d e^{-t \sqrt{\Delta+W}} u$ and $\phi_{2}:=e^{-t \sqrt{\vec{\Delta}+W}} d u$. Then

$$
\begin{aligned}
\int_{0}^{\infty}\left\|\phi_{1}\right\|_{L^{2}}^{2} d t & =\int_{0}^{\infty}\left\|d e^{-t \sqrt{\Delta+W}} u\right\|_{L^{2}}^{2} d t \\
& =\int_{0}^{\infty}\left\|d(\Delta+W)^{-1 / 2}(\Delta+W)^{1 / 2} e^{-t \sqrt{\Delta+W}} u\right\|_{L^{2}}^{2} d t \\
& \leq \int_{0}^{\infty}\left\|(\Delta+W)^{1 / 2} e^{-t \sqrt{\Delta+W}} u\right\|_{L^{2}}^{2} d t \\
& \leq \int_{0}^{1}\left\|(\Delta+W)^{1 / 2} u\right\|_{L^{2}}^{2} d t+\int_{1}^{\infty}\left\|t(\Delta+W)^{1 / 2} e^{-t \sqrt{\Delta+W}} u\right\|_{L^{2}}^{2} \frac{d t}{t^{2}} \\
& \leq\left\|(\Delta+W)^{1 / 2} u\right\|_{L^{2}}^{2}+\int_{1}^{\infty}\|u\|_{L^{2}}^{2} \frac{d t}{t^{2}} \\
& \leq C .
\end{aligned}
$$

Similarly

$$
\begin{aligned}
\int_{0}^{\infty}\left\|\partial_{t} \phi_{1}\right\|_{L^{2}}^{2} d t & =\int_{0}^{\infty}\left\|d \sqrt{\Delta+W} e^{-t \sqrt{\Delta+W}} u\right\|_{L^{2}}^{2} d t \\
& =\int_{0}^{\infty}\left\|d(\Delta+W)^{-1 / 2}(\Delta+W) e^{-t \sqrt{\Delta+W}} u\right\|_{L^{2}(X)}^{2} d t \\
& \leq \int_{0}^{\infty}\left\|(\Delta+W) e^{-t \sqrt{\Delta+W}} u\right\|_{L^{2}}^{2} d t \\
& \leq \int_{0}^{1}\|(\Delta+W) u\|_{L^{2}}^{2} d t+\int_{1}^{\infty}\left\|t^{2}(\Delta+W) e^{-t \sqrt{\Delta+W}} u\right\|_{L^{2}}^{2} \frac{d t}{t^{4}} \\
& \leq\|(\Delta+W) u\|_{L^{2}}^{2}+\int_{1}^{\infty}\|u\|_{L^{2}}^{2} \frac{d t}{t^{4}} \\
& \leq C
\end{aligned}
$$

and

$$
\begin{aligned}
\int_{0}^{\infty}\left\|\partial_{t}^{2} \phi_{1}\right\|_{L^{2}}^{2} d t & =\int_{0}^{\infty}\left\|d(\Delta+W) e^{-t \sqrt{\Delta+W}} u\right\|_{L^{2}}^{2} d t \\
& \leq\left\|(\Delta+W)^{3 / 2} u\right\|_{L^{2}}^{2}+\int_{1}^{\infty}\|u\|_{L^{2}}^{2} \frac{d t}{t^{6}} \\
& \leq C .
\end{aligned}
$$

By the same calculations, we can prove that $\phi_{2}, \partial_{t} \phi_{2}, \partial_{t}^{2} \phi_{2} \in L^{2}\left((0, \infty), L^{2}(X)\right)$. This shows that $\phi \in D\left(L_{1}\right) \cap D\left(L_{2}\right)$.

Step III. We prove that $d \Delta^{-\frac{1}{2}}$ is bounded on $L^{p}$. We write

$$
d \Delta^{-\frac{1}{2}}=\left(d \Delta^{-\frac{1}{2}}-d(\Delta+W)^{-\frac{1}{2}}\right)+d(\Delta+W)^{-\frac{1}{2}} .
$$

We have proved in the previous step that $d(\Delta+W)^{-\frac{1}{2}}$ is bounded on $L^{p}$ for all $p \in\left[2, p_{2}\right)$. Now we prove the boundedness of $d \Delta^{-\frac{1}{2}}-d(\Delta+W)^{-\frac{1}{2}}$. Following the ideas in [6, Section 3.6] 
with $A_{0}=\Delta+W$ and $A=\Delta$, we write

$$
\begin{aligned}
& d \Delta^{-\frac{1}{2}}-d(\Delta+W)^{-\frac{1}{2}} \\
& \quad=c \int_{0}^{\infty} t^{\frac{1}{2}} d\left(I+t A_{0}\right)^{-1} W(I+t A)^{-1} d t \\
& \quad=c \int_{0}^{\infty} d A_{0}^{-\frac{1}{2}}\left(t A_{0}\right)^{\frac{1}{2}}\left(I+t A_{0}\right)^{-\frac{1}{2}}\left(I+t A_{0}\right)^{-\frac{1}{2}} W^{\frac{1}{2}} W^{\frac{1}{2}}(I+t A)^{-1} d t .
\end{aligned}
$$

The operator $d A_{0}^{-\frac{1}{2}}=d(\Delta+W)^{-\frac{1}{2}}$ is bounded on $L^{p}$ for all $p \in\left[2, p_{2}\right)$. Next, $\left(t A_{0}\right)^{\frac{1}{2}}(I+$ $\left.t A_{0}\right)^{-\frac{1}{2}}$ is uniformly bounded (in $t>0$ ) on $L^{p}$ by the holomorphic functional calculus and the fact that $A_{0}$ has a Gaussian bound. For the last two terms in the previous integral, it suffices to prove that

$$
\int_{0}^{\infty}\left\|W^{\frac{1}{2}} e^{-s L}\right\|_{p \rightarrow p} \frac{d s}{\sqrt{s}} \leq C
$$

for all $p \in\left[2, p_{2}\right)$, where $L$ is $A_{0}$ or $A$. Noting that heat kernel of $A_{0}$ or $A$ satisfies Gaussian upper bound, so by volume condition (4.4) and doubling condition, we have for all $p \in\left[2, p_{2}\right)$ and $t>1$,

$$
\left\|\chi_{B\left(x_{0}, r_{0}\right)} e^{-t L}\right\|_{p \rightarrow \infty} \leq C_{x_{0}, r_{0}} t^{-\frac{p_{2}}{2 p}}
$$

Since $W \in C_{c}^{\infty}(M)$

$$
\int_{0}^{1}\left\|W^{\frac{1}{2}} e^{-s L}\right\|_{p \rightarrow p} \frac{d s}{\sqrt{s}} \leq C\|W\|_{\infty}^{\frac{1}{2}} \int_{0}^{1}\left\|e^{-s L}\right\|_{p \rightarrow p} \frac{d s}{\sqrt{s}} \leq C\|W\|_{\infty}^{\frac{1}{2}} \int_{0}^{1} \frac{d s}{\sqrt{s}} \leq C,
$$

and using supp $W \subset B\left(x_{0}, r_{0}\right)$, we deduce that for $p<p_{2}$

$$
\begin{aligned}
\int_{1}^{\infty}\left\|W^{\frac{1}{2}} e^{-s L}\right\|_{p \rightarrow p} \frac{d s}{\sqrt{s}} & \leq C\left\|W^{\frac{1}{2}}\right\|_{p} \int_{1}^{\infty}\left\|\chi_{B\left(x_{0}, r_{0}\right)} e^{-s L}\right\|_{p \rightarrow \infty} \frac{d s}{\sqrt{s}} \\
& \leq C\left\|W^{\frac{1}{2}}\right\|_{p} \int_{1}^{\infty} C_{x_{0}, r_{0}} s^{-\frac{p_{2}}{2 p}} \frac{d s}{\sqrt{s}} \leq C .
\end{aligned}
$$

For more details about this last step, we refer to [6, Section 3.6].

\section{Riesz transforms of Schrödinger operators}

In this section, we give some results on the boundedness of Riesz transforms $d A^{-\frac{1}{2}}$ of Schrödinger operators $A=\Delta+V$ with signed potential $V=V^{+}-V^{-}$.

We start with following result.

Theorem 5.1. Let $M$ be a complete non-compact Riemannian manifold satisfying assumptions (1.2) and (1.1) with doubling dimension D. Let $A$ be the Schrödinger operator with signed potential $V$ such that $V^{+} \in L_{\text {loc }}^{1}$ and $V^{-}$satisfies $\alpha$-subcritical condition (1.5). Then the associated Riesz transform $d A^{-\frac{1}{2}}$ is

i): bounded from $H_{A}^{1}(M)$ to $L^{1}\left(\Lambda^{1} T^{*} M\right)$,

ii): bounded from $H_{A}^{p}(M)$ to $L^{p}\left(\Lambda^{1} T^{*} M\right)$ for all $p \in[1,2]$,

iii): bounded on $L^{p}(M)$ for all $p \in(1,2]$ if $D \leq 2$ and all $p \in\left(p_{0}^{\prime}, 2\right]$ if $D>2$ where $p_{0}:=\frac{2 D}{(D-2)(1-\sqrt{1-\alpha})}$. 
Proof. Under these assumptions on $V$ it is proved in [6] (see the proof of estimate (43) in page 1127) that

$$
\left\|d A^{-\frac{1}{2}}\left(I-e^{-r^{2} A}\right)^{M} f\right\|_{L^{2}\left(U_{j}(B)\right)} \leq C 2^{-j s}\|f\|_{L^{2}(B)}
$$

for every ball $B$ with radius $r$ and for all $f \in L^{2}(M)$ with supp $f \subset B$. Here $M \geq 1, s>D / 2$ and $C>0$ are constants. Now by Lemma 3.2 we conclude that assertion i) holds.

Assertion ii) follows by interpolation between $H_{A}^{p}(M)$.

Assertion iii) follows from ii) by identifying $H_{A}^{p}(M)$ and $L^{p}$ (cf. Proposition 2.5) since the estimate $\left(D G_{p}\right)$ was proved in Theorem 3.4 in [6].

Note that assertion iii) of the previous theorem was already proved in [6].

For $p>2$, we give a consequence of Theorem 4.1 and [6, Theorem 3.9].

Theorem 5.2. Assume that the Riemannian manifold $M$ satisfies the doubling condition (1.1) and the heat kernel of the Laplacian satisfies the Gaussian upper bound (1.2). Assume also that the negative part of the Ricci curvature $R^{-}$satisfies (1.4) for some $p_{2}>3$. Let $A$ be the Schrödinger operator with signed potential $V$ which satisfies (1.6) and (1.5) for some $\alpha \in[0,1)$. Then $d A^{-\frac{1}{2}}$ is bounded on $L^{p}$ for $p_{0}^{\prime}<p<\frac{p_{0} r}{p_{0}+r}$ where $r=\inf \left(p_{1}, p_{2}\right)$.

This result is a combination of Theorem 4.1 and [6, Theorem 3.9]. Indeed it was proved in $\left[6\right.$, Theorem 3.9] that $\Delta^{\frac{1}{2}} A^{-\frac{1}{2}}$ is bounded on $L^{p}$ for $p_{0}^{\prime}<p<\frac{p_{0} r}{p_{0}+r}$ where $r=\inf \left(p_{1}, p_{2}\right)$ without assumptions on the Ricci curvature. By Theorem 4.1, $d \Delta^{-\frac{1}{2}}$ is bounded on $L^{p}$. The boundedness of $d A^{-\frac{1}{2}}$ follows by composition.

Remark 5.3. Suppose that $V^{-}=0$ (or equivalently $\alpha=0$ ) and $v(x, r) \geq C r^{D}$. Then $p_{0}=\infty$ and the assumptions in Theorem 5.2 hold if $R^{-} \in L^{\frac{D}{2}-\eta} \cap L^{\frac{D}{2}+\eta}$ and $V \in L^{\frac{D}{2}-\eta} \cap L^{\frac{D}{2}+\eta}$ for some $\eta>0$. Thus, the theorem gives that $d A^{-\frac{1}{2}}$ is bounded on $L^{p}$ for all $p \in(1, D)$. Note that one cannot expect a better interval for boundedness of the Riesz transform of Schrdinger operators as we will show in the next section.

\section{A negative Result for the Riesz transform of Schrödinger operators}

In this section, we show a negative result for the boundedness of the Riesz transform for Schrödinger operators. We prove even more : on a wide class of Riemannian manifolds, the Riesz transform $d(\Delta+V)^{-\frac{1}{2}}$ is never bounded on $L^{p}$ for any $p>D$, unless eventually $V=0$.

A result in this direction was given by Guillarmou and Hassel [19] on complete noncompact and asymptotically conic manifolds of dimension $n$. They assumed $V$ is non zero, smooth and sufficiently vanishing at infinity. They proved the Riesz transform $d(\Delta+V)^{-\frac{1}{2}}$ is not bounded on $L^{p}$ for $p>n$ if there exists a $L^{2}$ function $\psi$ such that $(-\Delta+V) \psi=0$.

For our concern, we recall that a Riemannian manifold $M$ satisfies the $L^{2}$ Poincaré inequality if there exists a constant $C>0$ such that for every $f \in W_{l o c}^{1,2}(M)$ and every ball $B=B(x, r)$

$$
\left(\int_{B}\left|f-f_{B}\right|^{2} d \mu\right)^{\frac{1}{2}} \leq C r\left(\int_{B}|d f|^{2} d \mu\right)^{\frac{1}{2}},
$$

where $f_{B}=\frac{1}{\mu(B)} \int_{B} f d \mu$. 
The main result of this section is the following theorem in which we consider for simplicity only non-negative potentials.

Theorem 6.1. Assume that $M$ satisfies the doubling volume condition (1.1) and the Poincaré inequality (6.1). Let $0 \leq V \in L_{\text {loc }}^{1}(M)$ and consider the Schrödinger operator $A=\Delta+V$. We suppose that there exists a positive function $\phi$ bounded on $M$ such that $e^{-t A} \phi=\phi$. If $\left\|d e^{-t A}\right\|_{p-p} \leq \frac{C}{\sqrt{t}}$ for some $p>\max (D, 2)$, then $V=0$. In particular, if $d A^{-\frac{1}{2}}$ is bounded on $L^{p}$ for some $p>\max (D, 2)$, then $V=0$.

Remark 6.2. The assumption $e^{-t A} \phi=\phi$ for all $t \geq 0$ with $\phi$ positive bounded was studied by several authors. We give here some references. In the Euclidean setting $M=\mathbb{R}^{n}$, Simon [26] proved that if the potential $V$ is in $L^{\frac{n}{2}-\eta} \cap L^{\frac{n}{2}+\eta}$ for a certain $\eta>0$, then the assumption $e^{-t A} \phi=\phi$ for all $t \geq 0$ is equivalent to the fact that $V^{-}$satisfies (1.5). With different methods, Grigor'yan [18] and Takeda [28] proved that if $M$ is non-parabolic and satisfies Li-Yau estimates and if the potential $V$ is nonnegative and Green-bounded on $M$, then such a function $\phi$ exists.

Proof of Theorem 6.1. From Lemma 6.3 below, we have for all $f \in W^{1, p}(M)$ and for almost every $x, x^{\prime} \in M$ with $\rho\left(x, x^{\prime}\right) \leq 1$

$$
\left|f(x)-f\left(x^{\prime}\right)\right| \leq C_{x, p}\|d f\|_{p} .
$$

Let $f \in C_{c}^{\infty}(M)$ and $x, x^{\prime} \in M$ with $\rho\left(x, x^{\prime}\right) \leq 1$ and fix $p>\max (D, 2)$ such that $\left\|d e^{-t A}\right\|_{p-p} \leq \frac{C}{\sqrt{t}}$ for all $t>0$. From (6.2), we have for all $t>0$

$$
\begin{aligned}
\left|e^{-t A}\left(v(., \sqrt{t})^{1-\frac{1}{p}} f\right)(x)-e^{-t A}\left(v(., \sqrt{t})^{1-\frac{1}{p}} f\right)\left(x^{\prime}\right)\right| & \leq C\left\|d e^{-t A} v(., \sqrt{t})^{1-\frac{1}{p}} f\right\|_{p} \\
& \leq \frac{C}{\sqrt{t}}\left\|e^{-\frac{t}{2} A} v(., \sqrt{t})^{1-\frac{1}{p}} f\right\|_{p} .
\end{aligned}
$$

Since (1.1) and (6.1) are equivalent to Li-Yau estimates (see [27]), the heat kernel $p_{t}(x, y)$ of $\Delta$ satisfies the Gaussian upper bound (1.2). Since $V \geq 0$, the heat kernel $k_{t}(x, y)$ of $A$ satisfies also the same Gaussian upper bound. As a consequence, the semigroup $e^{-t A}$ is uniformly bounded on $L^{1}(M)$ and the operator $e^{-t L} v(., \sqrt{t})$ is uniformly bounded from $L^{1}(M)$ to $L^{\infty}(M)$. An interpolation argument shows that for all $p \in[1, \infty]$ the operator $e^{-t L} v(., \sqrt{t})^{1-\frac{1}{p}}$ is bounded from $L^{1}(M)$ to $L^{p}(M)$ (see e.g. [8, Proposition 2.1.5]).

It follows from this and (6.3) that

$$
\left|e^{-t A}\left(v(., \sqrt{t})^{1-\frac{1}{p}} f\right)(x)-e^{-t A}\left(v(., \sqrt{t})^{1-\frac{1}{p}} f\right)\left(x^{\prime}\right)\right| \leq \frac{C}{\sqrt{t}}\|f\|_{1} .
$$

This extends by density to all $f \in L^{1}(M)$ and gives

$$
\left|\int_{M}\left(k_{t}(x, y)-k_{t}\left(x^{\prime}, y\right)\right) v(y, \sqrt{t})^{1-\frac{1}{p}} f(y) d \mu(y)\right| \leq \frac{C}{\sqrt{t}}\|f\|_{1} .
$$

Since the previous inequality is satisfied for all $f \in L^{1}(M)$, we obtain for a.e. $x, y \in M$ and all $t>0$

$$
\left|k_{t}(x, y)-k_{t}\left(x^{\prime}, y\right)\right| \leq \frac{C}{\sqrt{t} v(y, \sqrt{t})^{1-\frac{1}{p}}}
$$


Using (1.1) and (1.2) for $k_{t}(x, y)$ we find

$$
\begin{aligned}
& \left|k_{t}(x, y)-k_{t}\left(x^{\prime}, y\right)\right| \\
& \leq\left|k_{t}(x, y)-k_{t}\left(x^{\prime}, y\right)\right|^{\frac{1}{2}}\left[k_{t}(x, y)+k_{t}\left(x^{\prime}, y\right)\right]^{\frac{1}{2}} \\
& \leq \frac{C}{t^{\frac{1}{4}} v(y, \sqrt{t})^{\frac{1}{2}-\frac{1}{2 p}}}\left[\frac{C}{v(x, \sqrt{t})^{\frac{1}{2}}} \exp \left(-c \frac{\rho^{2}(x, y)}{t}\right)+\frac{C}{v\left(x^{\prime}, \sqrt{t}\right)^{\frac{1}{2}}} \exp \left(-c \frac{\rho^{2}\left(x^{\prime}, y\right)}{t}\right)\right] \\
& \leq \frac{C}{t^{\frac{1}{4}} v(y, \sqrt{t})^{1-\frac{1}{2 p}}}\left[\exp \left(-c \frac{\rho^{2}(x, y)}{t}\right)+\exp \left(-c \frac{\rho^{2}\left(x^{\prime}, y\right)}{t}\right)\right] .
\end{aligned}
$$

Therefore,

$$
\int_{M}\left|k_{t}(x, y)-k_{t}\left(x^{\prime}, y\right)\right| d \mu(y) \leq \frac{C}{t^{\frac{1}{4}}}\left[v(x, \sqrt{t})^{\frac{1}{2 p}}+v\left(x^{\prime}, \sqrt{t}\right)^{\frac{1}{2 p}}\right] .
$$

From (1.1) and since $\rho\left(x, x^{\prime}\right) \leq 1$, we deduce that for $t \geq 1$

$$
\int_{M}\left|k_{t}(x, y)-k_{t}\left(x^{\prime}, y\right)\right| d \mu(y) \leq \frac{C v(x, \sqrt{t})^{\frac{1}{2 p}}}{t^{\frac{1}{4}}} \leq \frac{C v(x, 1)^{\frac{1}{2 p}}}{t^{\frac{1}{4}\left(1-\frac{D}{p}\right)}} .
$$

Furthermore for all $t \geq 1$

$$
\begin{aligned}
\left|\phi(x)-\phi\left(x^{\prime}\right)\right| & =\left|e^{-t A} \phi(x)-e^{-t A} \phi\left(x^{\prime}\right)\right| \\
& =\left|\int_{M}\left(k_{t}(x, y)-k_{t}\left(x^{\prime}, y\right)\right) \phi(y) d \mu(y)\right| \\
& \leq \frac{C v(x, 1)^{\frac{1}{2 p}}}{t^{\frac{1}{4}\left(1-\frac{D}{p}\right)}}\|\phi\|_{\infty} .
\end{aligned}
$$

We let $t \rightarrow+\infty$ and since $p>D$ it follows that $\phi$ is constant on $M$. From the assumption $e^{-t A} \phi=\phi$ it follows that $A \phi=0$. The latter equality gives $V \phi=0$ and finally $V=0$ since $\phi$ is positive.

Finally, since the semigroup $e^{-t A}$ is analytic on $L^{p}$, if the Riesz transform $d A^{-\frac{1}{2}}$ is bounded on $L^{p}$ then

$$
\left\|d e^{-t A}\right\|_{p-p}=\left\|d A^{-\frac{1}{2}} A^{\frac{1}{2}} e^{-t A}\right\|_{p-p} \leq C\left\|A^{\frac{1}{2}} e^{-t A}\right\|_{p-p} \leq \frac{C^{\prime}}{\sqrt{t}} .
$$

The previous arguments show that $V=0$.

To complete the proof of the theorem, it remains to prove the following lemma.

Lemma 6.3. Let $p \geq 2$ and $p>D$. Assume that (1.1) and (6.1) are satisfied. For all $f \in W^{1, p}(M)$ and for almost every $x, x^{\prime} \in M$ with $\rho\left(x, x^{\prime}\right) \leq 1$ there exists a constant $C=C_{x, x^{\prime}, p}$ such that

$$
\left|f(x)-f\left(x^{\prime}\right)\right| \leq C\|d f\|_{p}
$$

Proof. The arguments in this proof are taken from [20, p. 13-14]. We repeat them for the reader's convenience. Write $B_{i}(x)=B\left(x, r_{i}\right)=B\left(x, \frac{\rho\left(x, x^{\prime}\right)}{2^{i}}\right)$ for each nonnegative integer $i$ and 
$f_{B}=\frac{1}{\mu(B)} \int_{B} f d \mu$. By the Lebesgue differentiation theorem we have for almost every $x \in M$, $f_{B_{i}(x)} \rightarrow f(x)$ as $i$ tends to infinity. Using (1.1), (6.1) and Hölder's inequality we obtain

$$
\begin{aligned}
\left|f(x)-f_{B_{0}(x)}\right| & \leq \sum_{i=0}^{\infty}\left|f_{B_{i+1}(x)}-f_{B_{i}(x)}\right| \\
& \leq \sum_{i=0}^{\infty} \frac{1}{\mu\left(B_{i+1}(x)\right)} \int_{B_{i+1}(x)}\left|f-f_{B_{i}(x)}\right| d \mu \\
& \leq C \sum_{i=0}^{\infty} \frac{1}{\mu\left(B_{i}(x)\right)} \int_{B_{i}(x)}\left|f-f_{B_{i}(x)}\right| d \mu \\
& \leq C \sum_{i=0}^{\infty}\left(\frac{1}{\mu\left(B_{i}(x)\right)} \int_{B_{i}(x)}\left|f-f_{B_{i}(x)}\right|^{2} d \mu\right)^{\frac{1}{2}} \\
& \leq C \sum_{i=0}^{\infty} \frac{\rho\left(x, x^{\prime}\right)}{2^{i}}\left(\frac{1}{\mu\left(B_{i}(x)\right)} \int_{B_{i}(x)}|d f|^{2} d \mu\right)^{\frac{1}{2}} \\
& \leq C \sum_{i=0}^{\infty} \frac{1}{2^{i} \mu\left(B_{i}(x)\right)^{\frac{1}{p}}}\|d f\|_{p} .
\end{aligned}
$$

Using property (1.1) and the fact that $\rho\left(x, x^{\prime}\right) \leq 1$, yields

$$
\frac{1}{\mu\left(B_{i}(x)\right)^{\frac{1}{p}}} \leq \frac{C 2^{i \frac{D}{p}}}{\rho\left(x, x^{\prime}\right)^{\frac{D}{p}} v(x, 1)^{\frac{1}{p}}} .
$$

Therefore, for $p>D$, we obtain

$$
\left|f(x)-f_{B_{0}(x)}\right| \leq C_{x, x^{\prime}, p}\|d f\|_{p} .
$$

Similarly

$$
\left|f\left(x^{\prime}\right)-f_{B_{0}\left(x^{\prime}\right)}\right| \leq C_{x, x^{\prime}, p}\|d f\|_{p} .
$$

Furthermore from the triangle inequality and (1.1) we have

$$
\begin{aligned}
\left|f_{B_{0}(x)}-f_{B_{0}\left(x^{\prime}\right)}\right| & \leq\left|f_{B_{0}(x)}-f_{2 B_{0}(x)}\right|+\left|f_{B_{0}\left(x^{\prime}\right)}-f_{2 B_{0}(x)}\right| \\
& \leq \frac{C}{\mu\left(2 B_{0}(x)\right)} \int_{2 B_{0}(x)}\left|f-f_{2 B_{0}(x)}\right| d \mu .
\end{aligned}
$$

We use the same arguments as above and obtain

$$
\left|f_{B_{0}(x)}-f_{B_{0}\left(x^{\prime}\right)}\right| \leq C_{x, x^{\prime}, p}\|d f\|_{p} .
$$

The lemma follows combining (6.8), (6.9) and (6.10).

\section{REFERENCES}

[1] P. Auscher, On necessary and sufficient conditions for $L^{p}$-estimates of Riesz transforms associated to elliptic operators on $\mathbb{R}$ and related estimates, Memoirs of the Amer. Math. Soc. 186 (2007), no. 871, xviii+75 pp.

[2] P. Auscher and T. Coulhon, Riesz transform on manifolds and Poincaré inequalities, Ann. Sc. Norm. Super. Pisa Cl. Sci. (5) 4 (2005), no. 3, 531-555. 
[3] P. Auscher, T. Coulhon, X. T. Duong and S. Hofmann, Riesz transform on manifolds and heat kernel regularity, Ann. Sci. Ecole Norm. Sup. (4) 37 (2004), no. 6, 911-957.

[4] P. Auscher, A. M Intosh and E. Russ, Hardy spaces of differential forms on Riemannian manifolds, $J$. Geom. Anal. 18 (2008), 192-248.

[5] P. Auscher, A. M $\mathrm{M}^{\mathrm{c}}$ Intosh and J.Morris, Calderón reproducing formulas and applications to Hardy spaces, 2013. Available at arXiv:1304.0168.

[6] J. Assaad and E. M. Ouhabaz, Riesz transforms of Schrödinger operators on manifolds, J. Geom. Anal. 22 (2012), 1108-1136.

[7] D. Bakry, Etude des transformations de Riesz dans les variétés riemanniennes à courbure de Ricci minorée, Séminaire de Probabilités, XXI, volume 1247 Lecture Notes in Math., 137-172. Springer, Berlin, 1987.

[8] S. Boutayeb, T. Coulhon and A. Sikora, A new approach to pointwise heat kernel upper bounds on doubling metric measure spaces, 2013. Available at arXiv:1311.0367v2.

[9] G. Carron, Riesz transforms on connected sums, Ann. Inst. Fourier 57 (2007), 2329-2343.

[10] G. Carron, Riesz transform on manifolds with quadratic curvature decay, 2014. Available at arXiv:1403.6278.

[11] T. Coulhon, Espaces de Lipschitz et inégalités de Poincaré, J. Funct. Anal. 136 (1996), 81-113.

[12] T. Coulhon and X. T. Duong, Riesz transform for $1 \leq p \leq 2$, Trans. Amer. Math. Soc. 351 (1999), 1151-1169.

[13] T. Coulhon and X. T. Duong, Riesz transform and related inequalities on noncompact Riemannian manifolds. Comm. Pure Appl. Math. 56 (2003), no. 12, 1728-1751.

[14] B. Devyver, A gaussian estimate for the heat kernel on differential forms and application to the Riesz transform, Math. Ann. 358 (2014), 25-68.

[15] X.T. Duong and L.X. Yan, Duality of Hardy and BMO spaces associated with operators with heat kernel bounds, J. Amer. Math. Soc. 18 (2005), 943-973.

[16] X.T. Duong and L.X. Yan, Spectral multipliers for Hardy spaces associated to non-negative self-adjoint operators satisfying Davies-Gaffney estimates, J. Math. Soc. Japan 63 (2011), 295-319.

[17] K. Engel and R. Nagel, One-Parameter Semigroups for Linear Evolution Equations, Graduate Texts in Mathematics, 194. Springer-Verlag, New York, 2000. xxii+586 pp. ISBN: 0-387-98463-1.

[18] A. Grigor'yan, Heat kernels on weighted manifolds and applications. The ubiquitous heat kernel, Contemp. Math., 398, Amer. Math. Soc., Providence, RI (2006), 93-191.

[19] C. Guillarmou and A. Hassell, Resolvent at low energy and Riesz transform for Schrödinger operators on asymptotically conic manifolds I, Math. Ann. 341 (2008), no. 4, 859-896

[20] P. Hajlasz and P. Koskela, Sobolev met Poincaré, Mem. Amer. Math. Soc. 145 (2000), no. 688, x+101 pp.

[21] S. Hofmann, G.Z. Lu, D. Mitrea, M. Mitrea and L.X. Yan, Hardy spaces associated to non-negative self-adjoint operators satisfying Davies-Gaffney estimates, Memoirs of Amer. Math. Soc. 214 (2011), no. 1007 , vi+78 pp.

[22] S. Hofmann and S. Mayboroda, Hardy and BMO spaces associated to divergence form elliptic operators, Math. Ann. 344 (2009), 37-116.

[23] S. Hofmann, S. Mayboroda and A. McIntosh, Second order elliptic operators with complex bounded measurable coefficients in $L^{p}$, Sobolev and Hardy spaces, Ann. Sci. École Norm. Sup. 44 (2011), 723800.

[24] J. Magniez, Riesz transforms of the Hodge-de Rham Laplacian on Riemannian manifolds, preprint 2014, available at http://arxiv.org/abs/1410.0034.

[25] A. Sikora, Riesz transform, Gaussian bounds and the method of wave equation, Math. Z. 247 (2004), 643-662.

[26] B. Simon, Brownian motion, $L^{p}$ properties of Schrödinger operators and the localization of binding, $J$. Funct. Anal. 35 (1980), 215-229

[27] L. Saloff-Coste, A note on Poincaré, Sobolev, and Harnack inequalities, Internat. Math. Res. Notices (1992), no. 2, 27-38. 
[28] M. Takeda, Gaussian bounds of heat kernels for Schrödinger operators on Riemannian manifolds, Bull. Lond. Math. Soc. 39 (2007), no. 1, 85-94.

[29] M. Uhl, Spectral multiplier theorems of Hörmander type via generalized Gaussian estimates, Ph.D. thesis, Jun 2011.

Peng Chen, Institut de Mathématiques de Bordeaux, Univ. Bordeaux, UMR 5251, 351, Cours de la Libération 33405 Talence, France

E-mail address: peng.chen@math.u-bordeaux1.fr

Jocelyn Magniez, Institut de Mathématiques de Bordeaux, Univ. Bordeaux, UMr 5251, 351, Cours de la Libération 33405 Talence, France

E-mail address: Jocelyn.Magniez@math.u-bordeaux1.fr

El Maati Ouhabaz, Institut de Mathématiques de Bordeaux, Univ. Bordeaux, UMR CNRS 5251, 351, Cours de la Libération 33405 Talence, France

E-mail address: Elmaati.Ouhabaz@math.u-bordeaux1.fr 